\title{
A Model for Stochastic Hybrid Systems with Application to Communication Networks ${ }^{\star \star}$
}

\author{
João P. Hespanha \\ Dept. of Electrical and Computer Eng., \\ Univ. of California, Santa Barbara, CA 92106-9560 \\ hespanha@ece.ucsb.edu
}

\begin{abstract}
We propose a model for Stochastic Hybrid Systems (SHSs) where transitions between discrete modes are triggered by stochastic events much like transitions between states of a continuoustime Markov chains. However, the rate at which transitions occur is allowed to depend both on the continuous and the discrete states of the SHS. Based on results available for Piecewise-Deterministic Markov Process (PDPs), we provide a formula for the extended generator of the SHS, which can be used to compute expectations and the overall distribution of the state.

As an application, we construct a stochastic model for on-off TCP flows that considers both the congestion-avoidance and slow-start modes and takes directly into account the distribution of the number of bytes transmitted. Using the tools derived for SHSs, we model the dynamics of the moments of the sending rate by an infinite system of ODEs, which can be truncated to obtain an approximate finite-dimensional model. This model shows that, for transfer-size distributions reported in the literature, the standard deviation of the sending rate is much larger than its average. Moreover, the later seems to vary little with the probability of packet drop. This has significant implications for the design of congestion control mechanisms.
\end{abstract}

keywords: Hybrid systems; Stochastic processes; Congestion control; TCP

\section{Introduction}

The idea of integrating logic and continuous dynamics in the control of complex systems is not new but only in the last 15 years has the theoretical analysis of hybrid systems (i.e., systems with both continuous dynamics and discrete logic) been pursued in a systematic fashion. A fair amount of research has been done on the modeling of hybrid systems $[1,3,10,12,29,30,36,40,44,46]$, but most of this work concentrated on deterministic systems.

Instances of stochastic hybrid systems can be found in the work on stochastic impulse control. For example Bensoussan and Lion [6], considered the problem of optimally choosing impulse times and intensities for a given stochastic differential equation. In more recent hybrid systems terminology, this would amount to optimally selecting reset times and the values to which the state is reset. One specific example is the classic optimal stopping time problem, where one wants to determine an optimal time instant at which the evolution of a differential equation is to be "frozen." Interestingly enough, Bensoussan and Lion [6] also considered the case of integer-valued states.

One of the more general formal models for stochastic hybrid systems was proposed by Hu et al. [20], where the deterministic differential equations for the continuous flows are replaced by their stochastic counterparts, and the reset maps are generalized to (state-dependent) distributions that define the probability density of the state after a discrete transition. However, in this model transitions are always triggered by deterministic conditions (guards) on the state.

\footnotetext{
* This material is based upon research supported by the National Science Foundation under Grants No. CCR0311084, ANI-0322476. Version dated March 27, 2005.

${ }^{\star \star}$ To appear in Nonlinear Analysis Special Issue on Hybrid Systems.
} 
In the model proposed in this paper for SHSs, transitions between modes are triggered by stochastic events, much like the transitions between states of a continuous-time Markov chains. However, the probability that a transition occurs in a given interval of time depends both on the continuous and discrete components of the current SHS's state. The state of the SHS so defined is a Piecewise-Deterministic Markov Process (PDP) in the sense of [14]. Based on this observation, we provide a formula for the extended generator of the SHS, which can be used to compute expectations and probability distributions/densities of its discrete/continuous states.

The basic model proposed is (i) limited to a deterministic evolution of the continuous state inside each discrete mode and (ii) it does not allow for transitions triggered by deterministic conditions on the state (e.g., guards being crossed). The first restriction is only introduced for simplicity of presentation and it is shown how the model can be generalized to allow for continuous evolutions driven by stochastic differential equations. The rationale for disallowing transitions triggered by guard-crossings is that this allows us to exclude Zeno phenomena [24, 46]. However, we show how one can approximate systems with guards (and potentially prone to Zeno phenomena) by a sequence of Zeno-free SHSs.

The model proposed is inspired by piecewise deterministic jump systems (PDJSs), where the evolution of the continuous state in each mode is modeled by a deterministic differential equation and transitions between modes are governed by a continuous-time Markov process [5, 13, 15, 16, 45]. In general, the transitions rates in PDJSs are assumed independent of the continuous state, which is too restrictive for our applications. The work of Filar et al. [16] is a notable exception but requires a time-scale separation between the (purely deterministic) continuous dynamics and the discrete jump dynamics. In switched diffusion processes (SDPs), as defined by Ghosh et al. [17], the evolution of the continuous state in each mode is modeled by a stochastic differential equation and transitions between modes are controlled by a continuous-time Markov process. The transition rates of the Markov process can depend on the state but transitions do not generate jumps on the continuous state (i.e., no resets). The reader is referred to [39] for a comparison of the models in [14, 17, 20]. The SHSs considered here can be viewed as special cases of general jump-diffusion processes [23]. In fact, Theorem 1 can be viewed as an Itô rule for SHSs. However, in the application of interest, we are faced with the technical difficulty that jump-intensities are not bounded and moreover the jump-distributions do not have compact support.

Our SHS model was inspired by the need to obtain accurate models for TCP congestion control in communication networks. The use of hybrid models to characterize the behavior of congestion control was proposed by Hespanha et al. [19] and further pursued in [8, 9]. In these models, packet drops trigger transitions between different "modes" for the evolution of TCP's congestion window size. The drop models in $[8,19]$ were completely deterministic but, as noted in [9], their use is limited to simple network topologies.

There is an extensive literature on models that describe the behavior of TCP congestion control for longlived flows, i.e., flows that have an infinite amount of data to transmit. A great deal of effort has been placed in characterizing the steady-state behavior of these flows [32, 33, 37, 38, 42]. In particular, in studying the relationship between the average transmission rate $\mu$, the average round-trip time $R T T$, and the per-packet drop rate $p_{\text {drop }}$ for a single TCP flow. In most of this work, $\mu$ and $p_{\text {drop }}$ should be understood as time-averages for a single TCP flow. This type of approach was also pursued in [25-28, 38, 41] to derive dynamic models for the congestion avoidance stage of long-lived TCP flows. However, these single-flow models are only valid over time scales much longer than the round-trip time for one packet. To avoid averaging over long time intervals, Misra et al. [34, 35] utilized ensemble averages to construct models for the dynamics of long-lived flows. Shakkottai and Srikant [41] also used stochastic aggregation to reduce the time-scales over which a single-flow model is valid. They showed that when $n$ single-flow models are aggregated, the average model becomes valid over time scales $n$ times smaller than those of the original single-flow models. Interestingly, aside from the different interpretation of the quantities involved, the ensemble-average models for long-lived flows do not differ significantly from the time-average ones.

We pursue here a stochastic analysis of the hybrid models proposed in [9]. As in [34, 35] time averaging is done over intervals of roughly one round-trip time to obtain continuously varying sending rates, and we then 
investigate the dynamics of ensemble averages. However, here we consider ensembles of on-off TCP flows. The off-periods are assumed exponentially distributed whereas the on-periods are determined by the amount of data being transfered. We take as given the probability distribution of the transfer-sizes, which implicitly determines the distribution for the on-periods. The model takes into account the correlation between the (variable) sending rate and the duration of the (also variable) on-periods. Not surprisingly, the ensemble behavior of on-off TCP flows varies very significantly with the probability distribution of the transfer sizes. By feeding our models with realistic distributions reported in the literature, we conclude that the dynamics of the sending rate is dominated by high-order statistics, exhibiting much larger standard deviations than the average value. Moreover, the packet drop rate seems to have a surprisingly small effect on the average drop rate but provides a strong control on its standard deviation. This has significant implications for the design of congestion control mechanisms.

To reach a wide audience we placed a great deal of effort in keeping technicalities to a minimum and relegated the most technical arguments to Sect. 3.3, which can be skipped without loss of continuity. The remainder of this paper is organized as follows: the basic stochastic hybrid model is introduced in Sect. 2 and several possible generalizations are discussed. Sect. 3 discusses the basic theoretical tools to analyze SHSs. In Sect. 4, we utilize SHSs to model TCP congestion control and illustrate how the tools derived in Sect. 3 can be used to analyze the resulting system. Sect. 5 contains some conclusions and directions for future research. The proofs of a few technical results are included in Appendix. A subset of the results in this paper were presented at the 2004 Workshop on Hybrid Systems : Computation and Control.

Notation. By a piecewise continuous signal it is meant a function $x:[0, \infty) \rightarrow \mathbb{R}^{n}$ that is right-continuous and has left-limit at every point. We denote by $x^{-}(t)$ the left-limit of $x(\tau)$ as $\tau \uparrow t$. A signal $x$ is called piecewise constant if it is piece-continuous and it is constant on every interval where it is continuous. Given a measurable space $(\Omega, \mathfrak{F})$ and probability measure $\mathrm{P}: \mathfrak{F} \rightarrow[0,1]$, vector-valued random variables $\boldsymbol{\alpha}: \Omega \rightarrow \mathbb{R}^{n}$ and stochastic processes $\mathbf{x}: \Omega \times[0, \infty) \rightarrow \mathcal{X} \subset \mathbb{R}^{n}$ are denoted in boldface. A stochastic process with piecewise constant sample paths is called a jump process. When a jump process takes values on the set $\mathbb{N}$ of nonnegative integers it is called a stochastic counter.

\section{Stochastic Hybrid Systems}

A stochastic hybrid system (SHS) is defined by a differential equation

$$
\dot{\mathbf{x}}=f(\mathbf{q}, \mathbf{x}, t), \quad f: \mathcal{Q} \times \mathbb{R}^{n} \times[0, \infty) \rightarrow \mathbb{R}^{n},
$$

a family of $m$ discrete transition/reset maps

$$
(\mathbf{q}, \mathbf{x})=\phi_{\ell}\left(\mathbf{q}^{-}, \mathbf{x}^{-}, t\right), \quad \phi_{\ell}: \mathcal{Q} \times \mathbb{R}^{n} \times[0, \infty) \rightarrow \mathcal{Q} \times \mathbb{R}^{n}, \ell \in\{1, \ldots, m\},
$$

and a family of $m$ transition intensities

$$
\lambda_{\ell}(\mathbf{q}, \mathbf{x}, t), \quad \lambda_{\ell}: \mathcal{Q} \times \mathbb{R}^{n} \times[0, \infty) \rightarrow[0, \infty), \ell \in\{1, \ldots, m\},
$$

where $\mathcal{Q}$ denotes a (typically finite) set with no particular topological structure. A SHS characterizes a jump process $\mathbf{q}: \Omega \times[0, \infty) \rightarrow \mathcal{Q}$ called the discrete state; a stochastic process $\mathbf{x}: \Omega \times[0, \infty) \rightarrow \mathbb{R}^{n}$ with piecewise continuous sample paths called the continuous state; and $m$ stochastic counters $\mathbf{N}_{\ell}: \Omega \times[0, \infty) \rightarrow \mathbb{N}$ called the transition counters.

In essence, between transition counter increments the discrete state remains constant whereas the continuous state flows according to (1); and at transition times the continuous and discrete states are reset according to (2). Each transition counter $\mathbf{N}_{\ell}$ counts the number of times that the corresponding discrete transition/reset map $\phi_{\ell}$ is "activated." The frequency at which this occurs is determined by the transition 
intensities (3). In particular, the probability that the counter $\mathbf{N}_{\ell}$ will increment and therefore that the corresponding transition takes place in an "elementary interval" $(t, t+d t]$ is given by $\lambda_{\ell}(\mathbf{q}(t), \mathbf{x}(t), t) d t$. In practice, one can think of the intensity of a transition as the instantaneous rate at which that transition occurs. We will shortly make these statements mathematically precise.

It is often convenient to represent SHSs by a directed graph as in Figure 1, where each vertex corresponds to a discrete mode and each edge to a transition between discrete modes. The vertices are labeled with the corresponding discrete mode and the vector fields that determines the evolution of the continuous state in that particular mode. The source of each edge is labeled with the probability that the transition will take place in an elementary interval $(t, t+d t]$ and the destination is labeled with the corresponding reset-map.

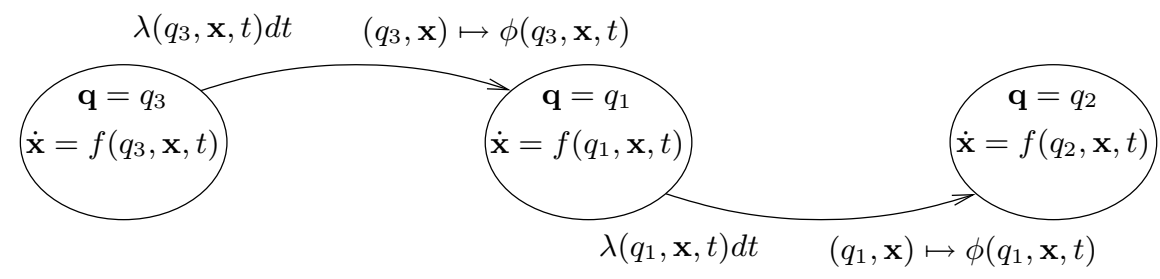

Fig. 1. Graphical representation of a stochastic hybrid system

\subsection{Construction of the stochastic processes}

We now provide the formal procedure to construct the sample paths of the discrete state, continuous state, and transition counters and show that it is indeed consistent with the intuition given above. Aside from its theoretical interest, this construction is useful to run Monte Carlo simulations of SHSs. It is inspired by the one used in [14, Chapter 2] to define Piecewise-Deterministic Markov Processes (PDPs). The following regularity assumption on the vector field $f$ is required for the SHS to be well defined:

Assumption 1. For every $\left(q_{0}, x_{0}, t_{0}\right) \in \mathcal{Q} \times \mathbb{R}^{n} \times[0, \infty)$ there exists a unique global solution $\varphi\left(\cdot ; t_{0}, q_{0}, x_{0}\right)$ : $\left[t_{0}, \infty\right) \rightarrow \mathbb{R}^{n}$ to (1) with initial condition $\mathbf{x}\left(t_{0}\right)=x_{0}$ and $\mathbf{q}\left(t_{0}\right)=q_{0}$.

In what follows, the $\boldsymbol{\mu}_{k}^{\ell}, \ell \in\{1, \ldots, m\}, k \in \mathbb{N}$ denote independent random variables all uniformly distributed in the interval $[0,1]$. We will call these transition triggers. Consider an initial condition $\left(q_{0}, x_{0}, t_{0}\right) \in \mathcal{Q} \times$ $\mathbb{R}^{n} \times[0, \infty)$. For a given $\omega \in \Omega$, the sample paths of $\mathbf{q}(\omega, \cdot):\left[t_{0}, \infty\right) \rightarrow \mathcal{Q}, \mathbf{x}(\omega, \cdot):\left[t_{0}, \infty\right) \rightarrow \mathbb{R}^{n}$, and all the $\mathbf{N}_{\ell}(\omega, \cdot):\left[t_{0}, \infty\right) \rightarrow \mathbb{N}$ can be constructed as follows:

1. Set $\mathbf{t}_{0}(\omega)=t_{0}, \mathbf{q}(\omega, 0)=q_{0}, \mathbf{x}(\omega, 0)=x_{0}, \mathbf{N}_{\ell}(\omega, 0)=0, \forall \ell$.

2. Let $\mathbf{t}_{1}(\omega)$ be the largest time on $\left(\mathbf{t}_{0}(\omega), \infty\right]$ for which

$$
e^{-\int_{\mathbf{t}_{0}(\omega)}^{t} \lambda_{\ell}\left(\mathbf{q}\left(\omega, \mathbf{t}_{0}(\omega)\right), \varphi\left(s ; \mathbf{t}_{0}(\omega), \mathbf{q}\left(\omega, \mathbf{t}_{0}(\omega)\right), \mathbf{x}\left(\omega, \mathbf{t}_{0}(\omega)\right)\right), s\right) d s}>\boldsymbol{\mu}_{0}^{\ell}(\omega),
$$

$\forall t \in\left[\mathbf{t}_{0}(\omega), \mathbf{t}_{1}(\omega)\right), \ell \in\{1, \ldots, m\}$.

3. On the interval $\left[\mathbf{t}_{0}(\omega), \mathbf{t}_{1}(\omega)\right)$, the sample paths of $\mathbf{q}(\omega, \cdot)$ and all the counters $\mathbf{N}_{\ell}(\omega, \cdot)$ remain constant, whereas the sample path of $\mathbf{x}(\omega, \cdot)$ equals $\varphi\left(\cdot ; \mathbf{t}_{0}(\omega), \mathbf{q}\left(\omega, \mathbf{t}_{0}(\omega)\right), \mathbf{x}\left(\omega, \mathbf{t}_{0}(\omega)\right)\right)$.

4. Denoting by $\ell_{1}(\omega) \in\{1,2, \ldots, m\}$ the index for which (4) is violated ${ }^{1}$ at time $t=\mathbf{t}_{1}(\omega)$, the counter $\mathbf{N}_{\ell_{1}(\omega)}(\omega)$ is incremented by one and

$$
\left(\mathbf{q}\left(\omega, \mathbf{t}_{1}(\omega)\right), \mathbf{x}\left(\omega, \mathbf{t}_{1}(\omega)\right)\right)=\phi_{\ell_{1}(\omega)}\left(\mathbf{q}^{-}\left(\omega, \mathbf{t}_{1}(\omega)\right), \mathbf{x}^{-}\left(\omega, \mathbf{t}_{1}\right), \mathbf{t}_{1}(\omega)\right)
$$

\footnotetext{
${ }^{1}$ In principle (4) could be violated simultaneously for more than one $\ell \in\{1, \ldots, m\}$, but since this event has zero probability we can ignore it.
} 
5. In case $\mathbf{t}_{1}(\omega)<\infty$, repeat the construction from the step 2 above with $\mathbf{t}_{0}(\omega), \boldsymbol{\mu}_{0}^{\ell}(\omega), \mathbf{t}_{1}(\omega), \boldsymbol{\ell}_{1}(\omega)$ replaced by $\mathbf{t}_{k}(\omega), \boldsymbol{\mu}_{k}^{\ell}(\omega), \mathbf{t}_{k+1}(\omega), \boldsymbol{\ell}_{k+1}(\omega)$, respectively, for $k=1,2, \ldots$

The random variables $\mathbf{t}_{k}$ defined by (4) are called transition times.

The stochastic processes so defined depend on the initial condition for the SHS. To emphasize this dependence we sometimes use $\mathrm{P}_{z_{0}}$ and $\mathrm{E}_{z_{0}}$ to denote the probability measure and expected value corresponding to the initial condition $z_{0}:=\left(q_{0}, x_{0}, t_{0}\right) \in \mathcal{Q} \times \mathbb{R}^{n} \times[0, \infty)$.

Step 1 provides the initialization for all the stochastic processes. Step 3 guarantees that the discrete state remains constant and the continuous state flows according to (1) between transitions. Step 4 enforces that the continuous and discrete states are reset according to (2) at transition times. The frequency at which these occur is determined by Step 2. In fact, one can derive from (4) (cf. Appendix) that

$$
\lim _{d t \downarrow 0} \frac{\mathrm{P}\left(\mathbf{N}_{\ell}(t+d t)>\mathbf{N}_{\ell}(t)\right)}{d t}=\lim _{d t \downarrow 0} \mathrm{E}\left[\frac{1}{d t} \int_{t}^{t+d t} \lambda_{\ell}(\mathbf{q}(t), \varphi(s ; t, \mathbf{q}(t), \mathbf{x}(t)), s) d s\right],
$$

which shows that the probability $\mathrm{P}\left(\mathbf{N}_{\ell}(t+d t)>\mathbf{N}_{\ell}(t)\right)$ that the transition $\phi_{\ell}$ will occur in an (arbitrarily) small interval $(t, t+d t]$ is proportional to the length of the interval with the proportionality constant given by the right-hand-side of (5). This equation specifies the precise meaning of the observation made above to the extent that "the probability that the counter $\mathbf{N}_{\ell}$ will increment in an elementary interval $(t, t+d t]$ is given by $\lambda_{\ell}(\mathbf{q}(t), \mathbf{x}(t), t) d t$." Note that when $\lambda_{\ell}$ is continuous, the right-hand-side of (5) is precisely equal to $\mathrm{E}\left[\lambda_{\ell}(\mathbf{q}(t), \mathbf{x}(t), t)\right]$.

The above construction guarantees that the sample-paths are indeed right-continuous and have left-limits at every point with probability one. However, without further assumptions there is no guarantee that the sample path are defined globally on $[0, \infty)$. We will return to this issue later.

\subsection{Generalizations}

The model for stochastic hybrid systems presented above is more general than it may appear at first. We discuss next some of the generalizations possible. We will return to these in Sect. 3.1 to study their implication on the results in Sect. 3.

The model allows for transitions where the next state is chosen according to a given distribution. For example, suppose one would like the intensity $\lambda(\mathbf{q}, \mathbf{x}, t)$ to trigger transitions to the discrete-states $q_{1}$ or $q_{2}$ with probabilities $p_{1}$ or $1-p_{1}$, respectively. This could be achieved by considering two transitions with intensities $p_{1} \lambda(\mathbf{q}, \mathbf{x}, t)$ and $\left(1-p_{1}\right) \lambda(\mathbf{q}, \mathbf{x}, t)$, respectively, and reset maps $\phi_{1}(q, x, t)=\left(q_{1}, \varphi_{1}(x, t), t\right)$ and $\phi_{2}(q, x, t)=\left(q_{2}, \varphi_{2}(x, t), t\right)$, respectively, where the $\varphi_{i}$ denote (possibly distinct) continuous-state resets.

The above model does not directly consider differential equations driven by stochastic processes. However, many important classes of stochastic processes can be obtained as the limit of jump processes that can be modeled by SHSs. For example, the stochastic differential equation

$$
\dot{\mathbf{x}}=a \mathbf{x}+b \dot{\mathbf{w}}
$$

where $\mathbf{w}$ denotes Brownian motion, can be regarded as the limit as $\epsilon \downarrow 0$ of the jump system with continuous dynamics $\dot{\mathbf{x}}=a \mathbf{x}$ and resets $\mathbf{x} \mapsto \mathbf{x}+b \sqrt{\epsilon}$ and $\mathbf{x} \mapsto \mathbf{x}-b \sqrt{\epsilon}$ both triggered with fixed intensity $\frac{1}{2 \epsilon}$. We can therefore model continuous evolutions of the form (6) as limits to a sequences of SHSs.

The basic model also does not directly consider discrete transitions triggered by deterministic conditions of the state, e.g., a guard being crossed. However, this behavior can also be obtained as the limiting solution to a sequence of SHSs. Consider for example the well known bouncing-ball single-mode deterministic hybrid system with dynamics $\ddot{x}=-g, g>0$ and state reset $(x, \dot{x}) \mapsto(0,-c \dot{x}), c \in(0,1)$ triggered by the condition 
$\dot{x}<0$ and $x \leq 0$. We could approximate this system by a sequence of SHSs for which the resets are triggered with intensities given by a "barrier function" of the form

$$
\lambda_{\epsilon}(x, \dot{x}):=\left\{\begin{array}{ll}
\epsilon e^{-x / \epsilon} & \dot{x}<0 \\
0 & \dot{x}>0,
\end{array}, \quad \epsilon>0 .\right.
$$

As $\epsilon \downarrow 0$, transitions will occur in a small neighborhood of $x=0$ with increasingly higher probability. Figure 2 shows confidence intervals for the sample-paths of three SHSs that approximate with increased accuracy the deterministic bouncing-ball. It is important to emphasize that, for any $\epsilon>0$, the sample paths of the SHSs are globally defined with probability one. This approach may in fact be a promising technique to overcome difficulties posed by the Zeno phenomena that occur for the deterministic bouncing-ball system [24, 46].
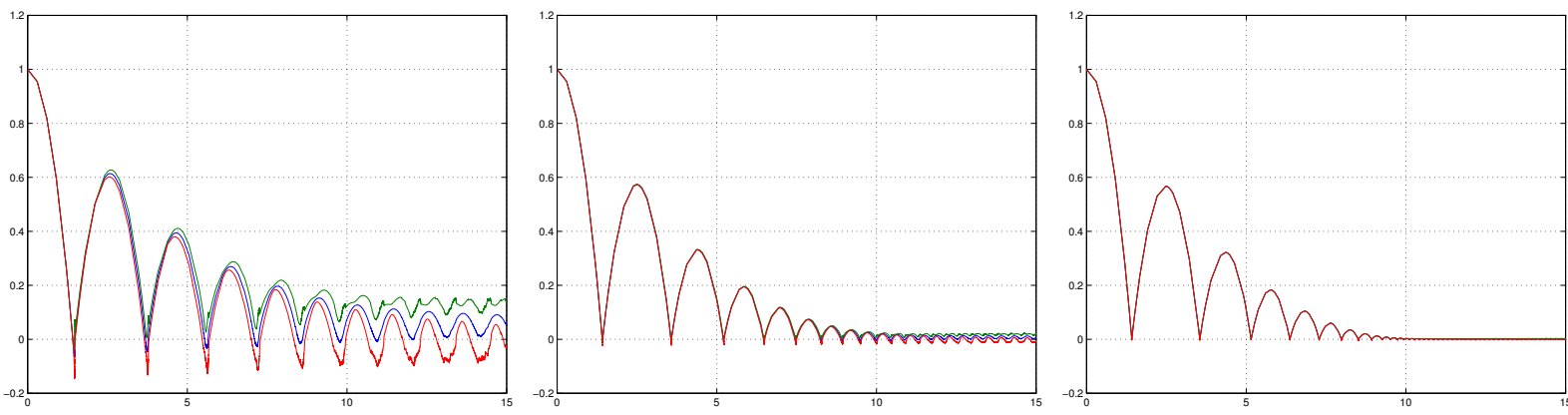

Fig. 2. 95\% confidence intervals for the $\mathbf{x}$ sample paths of three SHS that approximate the bouncing-ball deterministic system with Zeno time equal to 10 . The transition intensities of the SHSs are given by (7) with $\epsilon=10^{-2}, 10^{-3}, 10^{-4}$ from left to right. These results were obtained via Monte Carlo simulations.

\section{Generator for SHSs}

In this section we provide a result to compute expectations on the state of a SHS. The following assumptions are needed:

Assumption 2. (i) The transition intensities $\lambda_{\ell}: \mathcal{Q} \times \mathbb{R}^{n} \times[0, \infty) \rightarrow[0, \infty), \ell \in\{1, \ldots, m\}$ are measurable functions (e.g., continuous).

(ii) For every initial condition $z_{0}:=\left(q_{0}, x_{0}, t_{0}\right) \in \mathcal{Q} \times \mathbb{R}^{n}$ there exists a continuous functions $\alpha_{z_{0}}$ : $[0, \infty) \rightarrow[0, \infty)$ such that the sample-paths are defined globally and $\|\mathbf{x}(t)\| \leq \alpha_{z_{0}}(t), \forall t \geq t_{0}$ with probability one with respect to $\mathrm{P}_{z_{0}}$.

Assumption 2(ii) may be difficult to check, but we will shortly provide conditions that are more friendly to verify. We are now ready to state the main result of this section:

Theorem 1. Suppose that Assumptions 1 and 2 hold. For every initial condition $z_{0}:=\left(q_{0}, x_{0}, t_{0}\right) \in \mathcal{Q} \times$ $\mathbb{R}^{n} \times[0, \infty)$ and every function $\psi: \mathcal{Q} \times \mathbb{R}^{n} \times[0, \infty) \rightarrow \mathbb{R}$ that is continuously differentiable with respect to its second and third arguments, we have that

$$
\mathrm{E}_{z_{0}}[\psi(\mathbf{q}(t), \mathbf{x}(t), t)]=\psi\left(q_{0}, x_{0}, t_{0}\right)+\mathrm{E}_{z_{0}}\left[\int_{t_{0}}^{t}(L \psi)(\mathbf{q}(s), \mathbf{x}(s), s) d s\right],
$$

where $\forall(q, x, t) \in \mathcal{Q} \times \mathbb{R}^{n} \times[0, \infty)$

$$
(L \psi)(q, x, t):=\frac{\partial \psi(q, x, t)}{\partial x} f(q, x, t)+\frac{\partial \psi(q, x, t)}{\partial t}+\sum_{\ell=1}^{m}\left(\psi\left(\phi_{\ell}(q, x, t), t\right)-\psi(q, x, t)\right) \lambda_{\ell}(q, x, t),
$$


and $\frac{\partial \psi(q, x, t)}{\partial x}$ and $\frac{\partial \psi(q, x, t)}{\partial t}$ denote the gradient of $\psi(q, x, t)$ with respect to $x$ and the partial derivative of $\psi(q, x, t)$ with respect to $t$, respectively.

Following [14], we call the operator $\psi \mapsto L \psi$ defined by (9) the extended generator of the SHS. It is often convenient to write $(8)$ in the following differential form ${ }^{2}$

$$
\frac{\mathrm{d} \mathrm{E}[\psi(\mathbf{q}(t), \mathbf{x}(t), t)]}{\mathrm{d} t}=\mathrm{E}[(L \psi)(\mathbf{q}(t), \mathbf{x}(t), t)] .
$$

We leave the proof of Theorem 1 to Sect. 3.3 and proceed to contrast it with Itô calculus [22]. Consider the following jump-diffusion process

$$
\mathbf{d} \mathbf{x}=f(\mathbf{x}, t) d t+\sum_{\ell=1}^{m}\left(\phi_{\ell}(\mathbf{x}, t)-\mathbf{x}\right) d \mathbf{N}_{\ell}
$$

where the $\mathbf{N}_{\ell}$ are Poisson counters independent of each other and of the process' state $\mathbf{x}$. Each counter $\mathbf{N}_{\ell}$ has constant intensity $\lambda_{\ell}$ and induces a state jump $\mathbf{x} \mapsto \phi_{\ell}(\mathbf{x}, t)$ at increment-times. The Itô rule for (11) can be written as

$$
d \psi(\mathbf{x}, t)=\frac{\partial \psi(\mathbf{x}, t)}{\partial x} f(\mathbf{x}, t) d t+\frac{\partial \psi(\mathbf{x}, t)}{\partial t} d t+\sum_{\ell=1}^{m}\left(\psi\left(\phi_{\ell}(\mathbf{x}, t), t\right)-\psi(\mathbf{x}, t)\right) d \mathbf{N}_{\ell}
$$

where $\psi$ denotes a scalar-valued smooth function of $\mathbf{x}$ and $t$ (cf., e.g., [11]). The equation for the expectation of $\psi(\mathbf{x}, t)$ can be "derived" from (12) by replacing $d \mathbf{N}_{\ell}$ with $\lambda_{\ell} d t$, dividing the result by $d t$ and take expectations, which leads to

$$
\frac{\mathrm{d} \mathrm{E}[\psi(\mathbf{x})]}{\mathrm{d} t}=\mathrm{E}\left[\frac{\partial \psi(\mathbf{x}, t)}{\partial x} f(\mathbf{x}, t)+\frac{\partial \psi(\mathbf{x}, t)}{\partial t}+\sum_{\ell=1}^{m}\left(\psi\left(\phi_{\ell}(\mathbf{x}, t), t\right)-\psi(\mathbf{x}, t)\right) \lambda_{\ell}\right] .
$$

Theorem 1, thus suggests the following Itô rule for SHSs:

$$
d \psi(\mathbf{q}, \mathbf{x}, t)=\frac{\partial \psi(\mathbf{q}, \mathbf{x}, t)}{\partial x} f(\mathbf{q}, \mathbf{x}, t) d t+\frac{\partial \psi(\mathbf{q}, \mathbf{x}, t)}{\partial t} d t+\sum_{\ell=1}^{m}\left(\psi\left(\phi_{\ell}(\mathbf{q}, \mathbf{x}, t), t\right)-\psi(\mathbf{q}, \mathbf{x}, t)\right) d \mathbf{N}_{\ell}(\mathbf{q}, \mathbf{x})
$$

from which one would "derive" (10) using a procedure similar to the one described above for jump-diffusion processes. It is interesting to note that the existence of a discrete component $\mathbf{q}$ in the state does not significantly change the Itô rule.

Assumption 2(ii) rules out finite escape time almost surely. Although this is a mild requirement, it may be difficult to verify directly. The following lemma (proved in the Appendix) provides a condition that is more restrictive but easily checkable:

Lemma 1. Let $\phi_{\ell}^{x}: \mathcal{Q} \times \mathbb{R}^{n} \times[0, \infty) \rightarrow \mathbb{R}^{n}, \ell \in\{1, \ldots, m\}$ denote the projection of $\phi_{\ell}$ into $\mathbb{R}^{n}[$ i.e., $\phi_{\ell}^{x}(q, x, t)=\bar{x}$ where $\left.(\bar{q}, \bar{x})=\phi_{\ell}(q, x, t)\right]$. Assumptions 2(ii) holds, if there exists a continuous function $\gamma_{f}:[0, \infty) \rightarrow[0, \infty)$ and constants $c_{f}, c_{\phi}$ such that

$$
\|f(q, x, t)\| \leq \max \left\{\gamma_{f}(t)\|x\|, c_{f}\right\}, \quad \quad\left\|\phi_{\ell}^{x}(q, x, t)\right\| \leq \max \left\{\|x\|, c_{\phi}\right\},
$$

$\forall q \in \mathcal{Q}, x \in \mathbb{R}^{n}, t \geq 0, \ell \in\{1, \ldots, m\}$.

This lemma essentially requires $f$ and the $\phi_{\ell}$ to have linear growth in $x$ over $\mathbb{R}^{n}$. Moreover, the growth constant of the $\phi_{\ell}$ must not be larger than one. This is a strong requirement and one might be tempted to think that it could be replaced by a local condition if one would restrict one's attention to a finite time interval $[0, T], T<\infty$. It turns out that in general this is not true. Consider for example a SHS with a

\footnotetext{
${ }^{2}$ Recall that all signals are right-continuous with probability one and derivatives should be understood as right-limits.
} 
single discrete mode and scalar state $\mathbf{x} \in \mathbb{R}$ that evolves according to $\dot{\mathbf{x}}=0$, between transitions, and that undergoes a nonlocally Lipschitz reset $\mathbf{x} \mapsto \mathbf{x}^{p}, p>1$ at transition times. For simplicity, we consider a constant intensity $\mu$ for the transition. Given two time instants $t \geq \tau>0$, it is straightforward to conclude that $\mathbf{x}(t)=x_{0}^{p^{\mathbf{N}_{\ell}(t)}}$ and therefore

$$
\mathrm{E}_{x_{0}}[\mathbf{x}(t)]=\sum_{n=0}^{\infty} \frac{x_{0}^{p^{n}} \mu^{n} t^{n}}{n !} e^{-\mu t} .
$$

It turns out that when $x_{0}>1$ the above series does not converge for any $t>0$ and a "solution" to the SHS is not defined on any interval of positive length. Problems may still arise when the reset maps have linear growth but with a growth constant larger than one. For example, considering instead the globally Lipschitz reset map $\mathbf{x} \mapsto p \mathbf{x}, p>1$ and transition intensity $\lambda(\mathbf{x}, t)=x$, it is possible to show that $\mathrm{E}_{x_{0}}[\mathbf{x}(t)]$ would explode in finite time.

\subsection{Generalizations}

We now return to the generalizations of the basic SHS model mentioned in Sect. 2.2 and discuss their impact on Theorem 1. Suppose that we would like every intensity $\lambda_{\ell}$ to triggers a transition for which the next state is chosen according to a given distribution $\nu_{\ell}$ over $\mathcal{Q} \times \mathbb{R}^{n}$. Conceptually this can be viewed as expanding each original intensity $\lambda_{\ell}$ into a family of intensities, each one reseting to one particular next-state in $\mathcal{Q} \times \mathbb{R}^{n}$, and with intensity proportional to the value of the distribution $\nu_{\ell}$. In this case, the extended generator of the SHS becomes

$$
\begin{aligned}
(L \psi)(q, x, t):=\frac{\partial \psi(q, x, t)}{\partial x} f(q, x, t)+\frac{\partial \psi(q, x, t)}{\partial t} & \\
& +\sum_{\ell=1}^{m} \int_{\mathcal{Q} \times \mathbb{R}^{n}}(\psi(\bar{q}, \bar{x}, t)-\psi(q, x, t)) \lambda_{\ell}(q, x, t) \nu_{\ell}(q, x, t, d \bar{q}, d \bar{x}) .
\end{aligned}
$$

Needless to say that appropriate assumptions on the reset distributions $\nu_{\ell}$ are required to make sure that Assumption 2 holds.

Suppose now that one would like to drive the differential equation on each discrete mode by Gaussian white noise. In particular, suppose that one would like to replace (1) by

$$
\dot{\mathbf{x}}=f(\mathbf{q}, \mathbf{x}, t)+\sum_{i=1}^{k} g_{i}(\mathbf{q}, \mathbf{x}, t) \dot{\mathbf{w}}_{i},
$$

where each $\mathbf{w}_{i}$ denotes an independent Brownian motion process. The stochastic differential equation (13) can be regarded as the limit as $\epsilon \downarrow 0$ of the jump system with continuous dynamics $\dot{\mathbf{x}}=f(\mathbf{q}, \mathbf{x}, t)$ and resets $(q, x) \mapsto\left(q, x+\sqrt{\epsilon} g_{i}(q, x, t)\right),(q, x) \mapsto\left(q, x-\sqrt{\epsilon} g_{i}(q, x, t)\right)$ both triggered with fixed intensities $\frac{\epsilon}{2}$ (cf., e.g., $[11])$. As $\epsilon \downarrow 0$, the extended generator of the SHS converges to

$$
\begin{aligned}
(L \psi)(q, x, t):=\frac{\partial \psi(q, x, t)}{\partial x} f(q, x, t)+\frac{\partial \psi(q, x, t)}{\partial t}+\sum_{\ell=1}^{m}\left(\psi\left(\phi_{\ell}(q, x, t), t\right)-\psi(q, x, t)\right) \lambda_{\ell}(q, x, t)+ & \\
& +\frac{1}{2} \sum_{i=1}^{k} g_{i}(q, x, t)^{\prime} \frac{\partial^{2} \psi(q, x, t)}{\partial x^{2}} g_{i}(q, x, t)
\end{aligned}
$$

where $\frac{\partial^{2} \psi(q, x)}{\partial x^{2}}$ denotes the Hessian matrix of $\psi$ with respect to $x$ (cf. Appendix). Although, for each $\epsilon>0$, the assumptions of Lemma 1 are not satisfied for the reset maps above, the less strict assumptions of Lemma 2 are satisfied (at least for "well-behaved" $\psi$, e.g., bounded) and therefore the conclusions of Theorem 1 still hold (cf. Sect. 3.3 below). 


\subsection{Probability density}

Theorem 1 also allows one to compute the probability density function of the SHS's continuous state (assuming that one exists). The procedure is standard and is only included here for completeness.

Assume that there exists a joint probability density function $\rho(x, q ; t)$ such that, given any function $\psi: \mathcal{Q} \times \mathbb{R}^{n} \times[0, \infty) \rightarrow \mathbb{R}$ for which Theorem 1 holds, we have

$$
\mathrm{E}[\psi(\mathbf{q}(t), \mathbf{x}(t), t)]=\sum_{q \in \mathcal{Q}} \int_{\mathbb{R}^{n}} \psi(q, x, t) \rho(q, x ; t) d x .
$$

Then for a continuously differentiable function $\psi$ with bounded support, we conclude from Theorem 1 that

$$
\begin{aligned}
\sum_{q \in \mathcal{Q}} \int_{\mathbb{R}^{n}}\left(\psi(q, x, t) \frac{\partial \rho(q, x ; t)}{\partial t}-\frac{\partial \psi(q, x, t)}{\partial x}\right. & f(q, x, t) \rho(q, x ; t) \\
& \left.-\sum_{\ell=1}^{m}\left(\psi\left(\phi_{\ell}(q, x, t), t\right)-\psi(q, x, t)\right) \lambda_{\ell}(q, x, t) \rho(q, x ; t)\right) d x=0 .
\end{aligned}
$$

Assuming that $f(q, x, t) \rho(q, x ; t)$ is differentiable with respect to $x$ on the support of $\psi(q, \cdot, t)$, by integration by parts we obtain

$$
\begin{aligned}
\sum_{q \in \mathcal{Q}} \int_{\mathbb{R}^{n}} \psi(q, x, t)\left(\frac{\partial \rho(q, x ; t)}{\partial t}+\frac{\partial(f(q, x, t) \rho(q, x ; t))}{\partial x}\right. & \left.+\sum_{\ell=1}^{m} \lambda_{\ell}(q, x, t) \rho(q, x ; t)\right) d x \\
& =\sum_{q \in \mathcal{Q}} \int_{\mathbb{R}^{n}} \sum_{\ell=1}^{m} \psi\left(\phi_{\ell}(q, x, t), t\right) \lambda_{\ell}(q, x, t) \rho(q, x ; t) d x .
\end{aligned}
$$

In case $x \mapsto \phi_{\ell}^{x}(q, x, t)$ is invertible on $\mathbb{R}^{n}$ with inverse $\phi_{\ell}^{-x}: \mathcal{Q} \times \mathbb{R}^{n} \times[0, \infty) \rightarrow \mathbb{R}^{n}$, i.e., $\phi_{\ell}^{-x}\left(q, \phi_{\ell}^{x}(q, x, t), t\right)=$ $x, \forall q, x, t$, we can make the change of integration variable $z=\phi_{\ell}^{x}(q, x, t)$ on the right-hand-side and obtain

$$
\begin{aligned}
\sum_{q \in \mathcal{Q}} \int_{\mathbb{R}^{n}} \psi & (q, x, t)\left(\frac{\partial \rho(q, x ; t)}{\partial t}+\frac{\partial(f(q, x, t) \rho(q, x ; t))}{\partial x}+\sum_{\ell=1}^{m} \lambda_{\ell}(q, x, t) \rho(q, x ; t)\right) d x \\
= & \sum_{q \in \mathcal{Q}} \int_{\mathbb{R}^{n}} \sum_{\ell=1}^{m} \psi\left(\phi_{\ell}^{q}\left(q, \phi_{\ell}^{-x}(q, z, t), t\right), z\right) \lambda_{\ell}\left(q, \phi_{\ell}^{-x}(q, z, t), t\right) \rho\left(q, \phi_{\ell}^{-x}(q, z, t) ; t\right)\left|\frac{\partial \phi_{\ell}^{-x}(q, z, t)}{\partial z}\right| d z
\end{aligned}
$$

Choosing $\psi(q, x, t)$ to be zero for every $q$ other than $q_{0}$ and a delta-distribution around $x_{0}$, we obtain

$$
\begin{aligned}
\frac{\partial \rho\left(q_{0}, x_{0} ; t\right)}{\partial t}=-\frac{\partial\left(f\left(q_{0}, x_{0}, t\right) \rho\left(q_{0}, x_{0} ; t\right)\right)}{\partial x} & +\sum_{\ell=1}^{m}\left(-\lambda_{\ell}\left(q_{0}, x_{0}, t\right) \rho\left(q_{0}, x_{0} ; t\right)+\right. \\
& \left.+\sum_{q \in \mathcal{Q}_{\ell}\left[x_{0}, t\right]} \lambda_{\ell}\left(q, \phi_{\ell}^{-x}\left(q, x_{0}, t\right), t\right) \rho\left(q, \phi_{\ell}^{-x}\left(q, x_{0}\right) ; t\right)\left|\frac{\partial \phi_{\ell}^{-x}\left(q, x_{0}, t\right)}{\partial z}\right|\right),
\end{aligned}
$$

where $\mathcal{Q}_{\ell}\left[x_{0}, t\right]$ denotes the set of values $q \in \mathcal{Q}$ for which $\phi_{\ell}^{q}\left(q, \phi_{\ell}^{-x}\left(q, x_{0}, t\right)\right)=q_{0}$. When the previous system of partial differential equations (one for each $q_{0}$ ) has a unique solution, it defines the probability density of the SHS's state.

A similar derivation could have been done to obtain a Fokker-Planck-like equation for a SHS with a differential equation on each discrete mode driven by Brownian motion as in (13). In this case, using (14) instead of (9) we would eventually obtain 


$$
\begin{aligned}
& \frac{\partial \rho\left(q_{0}, x_{0} ; t\right)}{\partial t}=-\frac{\partial\left(f\left(q_{0}, x_{0}, t\right) \rho\left(q_{0}, x_{0} ; t\right)\right)}{\partial x}+\frac{1}{2} \sum_{i=1}^{k} \sum_{j} \sum_{l} \frac{\partial^{2}\left(g_{i}^{j}\left(q_{0}, x_{0}, t\right) g_{i}^{l}\left(q_{0}, x_{0}, t\right) \rho(q, x ; t)\right)}{\partial x_{l} \partial x_{j}} \\
& \quad+\sum_{\ell=1}^{m}\left(-\lambda_{\ell}\left(q_{0}, x_{0}, t\right) \rho\left(q_{0}, x_{0} ; t\right)+\sum_{q \in \mathcal{Q}_{\ell}\left[x_{0}, t\right]} \lambda_{\ell}\left(q, \phi_{\ell}^{-x}\left(q, x_{0}\right), t\right) \rho\left(q, \phi_{\ell}^{-x}\left(q, x_{0}\right) ; t\right)\left|\frac{\partial \phi_{\ell}^{-x}\left(q, x_{0}\right)}{\partial z}\right|\right),
\end{aligned}
$$

where $g_{i}^{j}$ denotes the $j$ th entry of the vector $g_{i}$.

\subsection{Proving Theorem 1}

Let $\mathbf{q}, \mathbf{x}, \mathbf{N}_{1}, \mathbf{N}_{2}, \ldots, \mathbf{N}_{m}$ be the stochastic processes characterized by a SHS using the construction in Sect. 2.1 with initial condition $\left(q_{0}, x_{0}, t_{0}\right) \in \mathcal{Q} \times \mathbb{R}^{n}$. The process $\mathbf{w}:=\left\{t, \mathbf{q}, \mathbf{x}, \mathbf{N}_{1}, \mathbf{N}_{2}, \ldots, \mathbf{N}_{m}\right\}$ is then a Piecewise-Deterministic Markov Processes (PDPs) as defined in [14, Chapter 2] with initial condition $\mathbf{w}(0)=\left\{t_{0}, q_{0}, x_{0}, 0, \ldots, 0\right\}$. This stems directly from the fact that the construction in Sect. 2.1 mimics the one in [14, Chapter 2] to define PDPs. By collecting results from [14] and adapting them to our setting the following is straightforward to prove.

Theorem 2. Suppose that Assumptions 1 and 2(i) hold and that, for every $z_{0}:=\left(q_{0}, x_{0}, t_{0}\right) \in \mathcal{Q} \times \mathbb{R}^{n}$,

$$
\mathrm{E}_{z_{0}}\left[\sum_{\ell} \mathbf{N}_{\ell}(t)\right]<\infty, \quad \forall t \geq t_{0}
$$

Then (8) holds for every $z_{0}:=\left(q_{0}, x_{0}, t_{0}\right) \in \mathcal{Q} \times \mathbb{R}^{n}$ and every function $\psi: \mathcal{Q} \times \mathbb{R}^{n} \times[0, \infty) \rightarrow \mathbb{R}$ that is continuously differentiable with respect to its second argument and

$$
\mathrm{E}_{z_{0}}\left[\sum_{\mathbf{t}_{k} \leq t} \mid \psi\left(\mathbf{q}\left(\mathbf{t}_{k}\right), \mathbf{x}\left(\mathbf{t}_{k}\right), \mathbf{t}_{k}\right)-\psi\left(\mathbf{q}\left(\mathbf{t}_{k}^{-}\right), \mathbf{x}\left(\mathbf{t}_{k}^{-}\right), \mathbf{t}_{k}\right)\right]<\infty, \quad \forall t \in \mathbb{N} .
$$

Proof of Theorem 2. Assumptions 1, 2(i), and (15) guarantee that the standard conditions required by [14, Theorem 26.14] hold for the PDP defined by $\mathbf{w}:=\left\{t, \mathbf{q}, \mathbf{x}, \mathbf{N}_{1}, \ldots, \mathbf{N}_{m}\right\}^{3}$. Moreover, (16) guarantees that $\bar{\psi}\left(t, q, x, n_{1}, \ldots, n_{m}\right):=\psi(q, x, t)$ belongs to the domain of the extended generator of $\mathbf{w}$ and also that

$$
\mathbf{C}^{\psi}(t):=\psi(\mathbf{q}(t), \mathbf{x}(t), t)-\psi\left(q_{0}, x_{0}, t_{0}\right)-\int_{t_{0}}^{t}(L \psi)(\mathbf{q}(s), \mathbf{x}(s)) d s
$$

is actually a Martingale rather than just a local Martingale (cf., [14, Remark 26.14]). Therefore

$$
\mathrm{E}_{z_{0}}\left[\mathbf{C}^{\psi}(t)\right]=\mathbf{C}^{\psi}\left(t_{0}\right)=0
$$

from which (8) follows.

In view of Theorem 2, to prove Theorem 1 it suffices to show that Assumption 2(ii) implies that both (15) and (16) hold. Actually, as shown in the following Lemma, something slightly weaker than Assumption 2(ii) suffices.

Lemma 2. For a given $z_{0}:=\left(q_{0}, x_{0}, t_{0}\right) \in \mathcal{Q} \times \mathbb{R}^{n}$ and function $\psi: \mathcal{Q} \times \mathbb{R}^{n} \rightarrow \mathbb{R}$, assume that there exist continuous functions $\alpha, \beta:[0, \infty) \rightarrow[0, \infty)$ such that

$$
\lambda_{\ell}(\mathbf{q}(t), \mathbf{x}(t), t) \leq \alpha(t), \quad|\psi(\mathbf{q}(t), \mathbf{x}(t), t)| \leq \beta(t), \quad \forall \ell, t \geq 0,
$$

with probability one with respect to $\mathrm{P}_{z_{0}}$. Then (15) and (16) hold.

\footnotetext{
${ }^{3}$ At every switching time at least one of the counters $\mathbf{N}_{\ell}$ increases by one so assumption $24.8,3$. of [14] holds trivially.
} 
Before proving Lemma 2 note that Assumption 2(ii) does guarantee the existence of the functions $\alpha$ and $\beta$ for which (17) holds and therefore Theorem 1 follows directly from Theorem 2 and Lemma 2.

Proof of Lemma 2. From the definition of the transition times $\mathbf{t}_{k}$, we have that

$$
\begin{aligned}
\mathrm{P}\left(\sum_{\ell} \mathbf{N}_{\ell}(t) \geq k\right)=\mathrm{P}\left(\mathbf{t}_{k}\right. & \leq t)=\mathrm{P}\left(e^{-\int_{\mathbf{t}_{k-1}}^{t} \lambda_{\ell_{k}}(\mathbf{q}(s), \mathbf{x}(s), s) d s} \leq \boldsymbol{\mu}_{k-1}^{\ell_{k}}\right)= \\
& =\mathrm{P}\left(e^{-\int_{\mathbf{t}_{k-1}}^{t} \lambda_{\ell_{k}}(\mathbf{q}(s), \mathbf{x}(s), s) d s} \leq \boldsymbol{\mu}_{k-1}^{\boldsymbol{\ell}_{k}}, e^{-\int_{\mathbf{t}_{i-1}}^{\mathbf{t}_{i}} \lambda_{\ell_{i}}(\mathbf{q}(s), \mathbf{x}(s), s) d s} \leq \boldsymbol{\mu}_{i-1}^{\boldsymbol{\ell}_{i}}, \forall i<k\right) .
\end{aligned}
$$

Note that all the events added in the last equality occur with probability one (actually with equality) so they do not change the overall probability. Because of (17),

$$
e^{-\int_{a}^{b} \lambda_{\ell_{k}}(\mathbf{q}(s), \mathbf{x}(s), s) d s} \geq e^{-\int_{a}^{b} \alpha(s) d s}, \quad \forall b>a \geq 0,
$$

with probability one, therefore

$$
\begin{aligned}
& \mathrm{P}\left(\sum_{\ell} \mathbf{N}_{\ell}(t) \geq k\right) \leq \mathrm{P}\left(e^{-\int_{\mathbf{t}_{k-1}}^{t} \alpha(s) d s} \leq \boldsymbol{\mu}_{k-1}^{\ell_{k}}, e^{-\int_{\mathbf{t}_{i-1}}^{\mathbf{t}_{i}} \alpha(s) d s} \leq \boldsymbol{\mu}_{i-1}^{\ell_{i}}, \forall 1 \leq i<k\right) \\
& \quad=\mathrm{P}\left(\int_{\mathbf{t}_{k-1}}^{t} \alpha(s) d s \geq-\log \boldsymbol{\mu}_{k-1}^{\ell_{k}}, \int_{\mathbf{t}_{i-1}}^{\mathbf{t}_{i}} \alpha(s) d s \geq-\log \boldsymbol{\mu}_{i-1}^{\ell_{i}}, \forall 1 \leq i<k\right) \\
& \quad \leq \mathrm{P}\left(\int_{\mathbf{t}_{k-1}}^{t} \alpha(s) d s+\sum_{i=1}^{k-1} \int_{\mathbf{t}_{i-1}}^{\mathbf{t}_{i}} \alpha(s) d s \geq-\sum_{i=1}^{k} \log \boldsymbol{\mu}_{i-1}^{\boldsymbol{\ell}_{i}}\right) \\
& \quad=\mathrm{P}\left(\int_{t_{0}}^{t} \alpha(s) d s \geq-\sum_{i=1}^{k} \log \boldsymbol{\mu}_{i-1}^{\boldsymbol{\ell}_{i}}\right) .
\end{aligned}
$$

Since all the $\boldsymbol{\mu}_{i-1}^{\ell_{i}}$ are independent and uniformly distributed in the interval [0,1], the $-\log \boldsymbol{\mu}_{i-1}^{\ell_{i}}$ are independent random variables exponentially distributed with unit mean. Therefore $-\sum_{i=1}^{k} \log \boldsymbol{\mu}_{i-1}^{\boldsymbol{\ell}_{i}}$ is Erlang distributed and

$$
\mathrm{P}\left(-\sum_{i=1}^{k} \log \boldsymbol{\mu}_{i-1}^{\ell_{i}} \leq \eta\right)=1-\sum_{i=0}^{k-1} e^{-\eta} \frac{\eta^{i}}{i !} \leq \frac{\eta^{k}}{k !}, \quad \forall \eta \geq 0
$$

where the right-inequality can be proved by showing that

$$
\varphi(\eta):=1-\sum_{i=0}^{k-1} e^{-\eta} \frac{\eta^{i}}{i}-\frac{\eta^{k}}{k}
$$

is zero at zero and $\frac{\partial \varphi(\eta)}{\partial \eta}=\frac{\eta^{k-1}}{(k-1)}\left(e^{-\eta}-1\right)<0, \forall \eta>0$. We therefore conclude that

$$
\mathrm{P}\left(\sum_{\ell} \mathbf{N}_{\ell}(t) \geq k\right) \leq \frac{\left(\int_{t_{0}}^{t} \alpha(s) d s\right)^{k}}{k !}
$$

Finally,

$$
\mathrm{E}\left[\sum_{\ell} \mathbf{N}_{\ell}(t)\right] \leq \sum_{k=0}^{\infty} k \mathrm{P}\left(\sum_{\ell} \mathbf{N}_{\ell}(t) \geq k\right) \leq \sum_{k=0}^{\infty} k \frac{\left(\int_{t_{0}}^{t} \alpha(s) d s\right)^{k}}{k !}=\left(\int_{t_{0}}^{t} \alpha(s) d s\right) e^{\int_{t_{0}}^{t} \alpha(s) d s}<\infty,
$$

from which (15) follows. On the other hand,

$$
\mathrm{E}\left[\sum_{\mathbf{t}_{k} \leq t}\left|\psi\left(\mathbf{q}\left(\mathbf{t}_{k}\right), \mathbf{x}\left(\mathbf{t}_{k}\right), \mathbf{t}_{k}\right)-\psi\left(\mathbf{q}\left(\mathbf{t}_{k}^{-}\right), \mathbf{x}\left(\mathbf{t}_{k}^{-}\right), \mathbf{t}_{k}\right)\right|\right] \leq \mathrm{E}\left[\sum_{\mathbf{t}_{k} \leq t} 2 \beta(t)\right]=\mathrm{E}\left[2 \beta(t) \sum_{\ell} \mathbf{N}_{\ell}(t)\right]
$$

where, without loss of generality, we assumed that $\beta$ is monotone increasing. Inequality (16) follows from this and (15). 


\section{A stochastic model for TCP flows}

In this section we present a SHSs model for a single-user on-off TCP flow based on the hybrid modeling framework proposed by Bohacek et al. [9]. The model is represented graphically in Figure 3. It has two con-

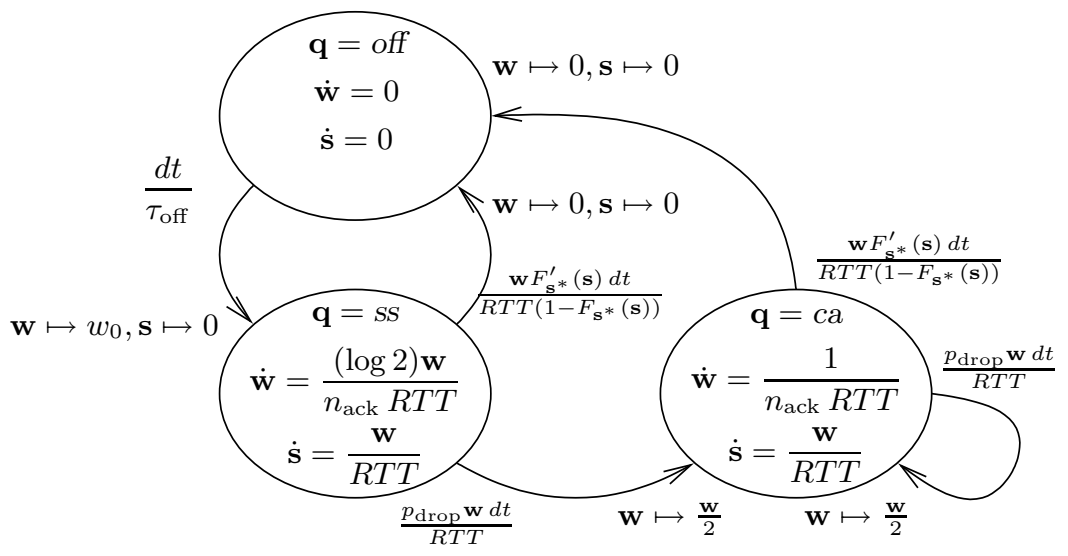

Fig. 3. Stochastic hybrid model for a TCP flow, where $n_{\text {ack }}$ denotes the number of data packets acknowledged per each ACK packet received and $w_{0}:=.693$ when $n_{\text {ack }}=1$ or $w_{0}:=1.428$ when $n_{\text {ack }}=2$.

tinuous states - TCP's congestion window size w and the cumulative number of packets sent in a particular connection $\mathbf{s}$ - and three discrete states $\{$ off, ss, ca $\}$.

1. During the off mode the flow is inactive and we simply have $\mathbf{w}=\mathbf{s}=0$.

2. The ss mode corresponds to TCP's slow-start. In this mode, the congestion window size w increases by one for each ACK packet received and $\mathbf{w}$ packets are sent each round-trip time $R T T$. This can be modeled by

$$
\dot{\mathbf{w}}=\frac{(\log 2) \mathbf{r}}{n_{\text {ack }}}=\frac{(\log 2) \mathbf{w}}{n_{\text {ack }} R T T}, \quad \dot{\mathbf{s}}=\mathbf{r}=\frac{\mathbf{w}}{R T T}
$$

where $\mathbf{r}=\frac{\mathbf{w}}{R T T}$ denotes the instantaneous average sending rate and $n_{\text {ack }}$ the number of data packets acknowledged per each ACK packet received. The ( $\log 2)$ term compensates for the error introduced by approximating the discrete increments by a continuous increase. Indeed, without delayed ACKs we have $n_{\text {ack }}=1$ and this model leads to the usual doubling of $\mathbf{w}$ every $R T T$. With delayed ACKs, typically $n_{\text {ack }}=2$ and this model leads to a multiplication of the $\mathbf{w}$ by $\sqrt{2}$ every $R T T$. This is consistent with the analysis by Sikdar et al. [43], which shows that for $n_{\text {ack }}=2$ the number of packets sent in the $n$th round-trip time of slow-start is approximately equal to $\left(1+\frac{\sqrt{2}}{2}\right) \sqrt{2}^{n}$. This formula is matched exactly by the fluid model in (18) when one sets $\mathbf{w}=1.428$ at the beginning of ss. On the other hand, for $n_{\text {ack }}=1$, the number of packets sent in the $n$th round-trip time of slow-start should be equal to $2^{n-1}$. This matches the fluid model by making $\mathbf{w}=.693$ at the beginning of ss.

3 . The ca mode corresponds to TCP's congestion-avoidance. In this mode $\mathbf{w}$ increases by $1 / \mathbf{w}$ for each ACK packet received and, as in slow-start, w packets are sent each round-trip time RTT. This can be modeled by

$$
\dot{\mathbf{w}}=\frac{1}{\mathbf{w}} \frac{\mathbf{r}}{n_{\text {ack }}}=\frac{1}{n_{\text {ack }} R T T}, \quad \dot{\mathbf{s}}=\mathbf{r}=\frac{\mathbf{w}}{R T T}
$$

The transitions between modes occur as follows:

1. Drops occurrences - which correspond to transitions from the ss or the ca modes to the ca mode - occur at a rate $p_{\text {drop }} \mathbf{r}$, where $p_{\text {drop }}$ denotes the per-packet drop probability and $\mathbf{r}:=\frac{\mathbf{w}}{R T T}$ the packet sending 
rate. The corresponding intensity and reset maps are given by

$$
\begin{aligned}
\lambda_{\text {drop }}(q, w, s, t) & := \begin{cases}\frac{p_{\mathrm{drop}}(t) w}{R T T} & q \in\{\mathrm{ss}, \mathrm{ca}\} \\
0 & \text { otherwise }\end{cases} \\
\phi_{\text {drop }}(q, w, s, t) & := \begin{cases}\left(c \mathrm{ca}, \frac{w}{2}, s\right) & q \in\{\mathrm{ss}, \mathrm{ca}\} \\
(q, w, s) & \text { otherwise. }\end{cases}
\end{aligned}
$$

2. The start of new flows - which correspond to the transitions from the off to the ss mode - occur at a rate $\frac{1}{\tau_{\text {off }}}$. This is consistent with an exponentially distributed duration of the off periods with average $\tau_{\text {off }}$. The corresponding intensity and reset maps are given by

$$
\begin{aligned}
\lambda_{\text {start }}(q, w, s, t) & := \begin{cases}\frac{1}{\tau_{\text {off }}} & q=\text { off } \\
0 & \text { otherwise }\end{cases} \\
\phi_{\text {start }}(q, w, s, t) & := \begin{cases}\left(s s, w_{0}, 0\right) & q=\text { off } \\
(q, w, s) & \text { otherwise }\end{cases}
\end{aligned}
$$

where

$$
w_{0}:= \begin{cases}.693 & n_{\text {ack }}=1 \\ 1.428 & n_{\text {ack }}=2,\end{cases}
$$

3. The termination of flows - which correspond to transitions from the ss and ca modes to the off modeoccur at a rate

$$
\frac{\mathbf{r} F_{\mathbf{s}^{*}}^{\prime}(\mathbf{s})}{1-F_{\mathbf{s}^{*}}(\mathbf{s})}, \quad \mathbf{r}:=\frac{\mathbf{w}}{R T T}
$$

This is consistent with a distribution $F_{\mathbf{s}^{*}}:[0, \infty) \rightarrow[0,1]$ for the number $\mathbf{s}^{*}$ of packets sent in each TCP session (cf. Appendix). The corresponding intensity and reset maps are given by

$$
\begin{aligned}
\lambda_{\text {end }}(q, w, s, t) & := \begin{cases}\frac{w F_{\mathrm{s}^{*}}^{\prime}(s)}{R T T\left(1-F_{\mathbf{s}^{*}}(s)\right)} & q \in\{\text { ss }, c a\} \\
0 & \text { otherwise }\end{cases} \\
\phi_{\text {end }}(q, w, s, t) & := \begin{cases}(o f f, 0,0) & q \in\{\text { ss }, c a\} \\
(q, w, s) & \text { otherwise }\end{cases}
\end{aligned}
$$

Three main simplifications were considered: we ignored fast-recovery after a drop is detected by three duplicate ACKs, we ignored the delay between the time a drop takes place and the time it is detected, and we ignored timeouts. Fast-recovery takes relatively little time and has little impact on the overall throughput unless the number of drops is very high [8]. Timeouts can have a severe impact on the throughput when drops are highly correlated. Here, we are mostly interested in RED for which high correlations are unlikely. The drop detection delay is mostly important for the stability analysis of congestion control protocols, which will not be pursued here. We focus our attention on two specific instances of the general model in Figure 3:

Exponential sizes This model is obtained by assuming that the number of packets to transmit is exponentially distributed with mean $k$, i.e., $F_{\mathbf{s}^{*}}(s)=1-e^{-\frac{s}{k}}, \forall s \geq 0$. In this case, the intensity of the counter $\mathbf{N}_{\text {end }}$ is given by

$$
\lambda_{\text {end }}(q, w, s, t):= \begin{cases}\frac{k^{-1} w}{R T T} & q \in\{\text { ss }, \text { ca }\} \\ 0 & \text { otherwise. }\end{cases}
$$


Mixed-exponential sizes It has been observed that modeling the distribution of transfer-sizes as an exponential is an over-simplification. For example, it has been observed that heavy-tail models are more fitting to experimental data (cf., e.g., $[2,4]$ ). An alternative that turns out to be computationally attractive and still fits reasonably well with experimental data is a mixture of exponentials. According to this model, transfer-sizes are sampled from a family of $M$ exponential random variables $\mathbf{s}_{i}, i \in\{1,2, \ldots, M\}$ by selecting a sample from the $i$ th random variable $\mathbf{s}_{i}$ with probability $p_{i}$. Each $\mathbf{s}_{i}$ corresponds to a distinct mean transfer-size $k_{i}$. To model this as a SHS, we consider $M$ alternative $\left\{\mathrm{ss}_{i}, c \mathrm{ca}_{i}: i=1,2, \ldots, M\right\}$ modes, each corresponding to a specific exponential distribution for the transfer-sizes. The transition from the inactive mode off to the slow-start mode $s_{i}$ corresponding to a mean transfer-size of $k_{i}$ occurs with probability $p_{i}$ and are associated with intensities and reset maps given by

$$
\lambda_{i}(q, w, s, t):=\left\{\begin{array}{ll}
\frac{p_{i}}{\tau_{\text {off }}} & q=\text { off } \\
0 & \text { otherwise }
\end{array} \quad \phi_{i}(q, w, s, t):= \begin{cases}\left(\mathrm{ss}_{i}, w_{0}, 0\right) & q=\text { off } \\
(q, w, s) & \text { otherwise }\end{cases}\right.
$$

which replace the $\lambda_{\text {start }}$ and $\phi_{\text {start }}$ in the previous model. To obtained the desired distribution for the transfersize, the intensity and reset maps of the transitions to the inactive mode off, should be replaced by

$$
\begin{aligned}
\lambda_{\text {end }}(q, w, s, t) & := \begin{cases}\frac{k_{1}^{-1} w}{R T T} & q \in\left\{\mathrm{ss}_{1}, \mathrm{ca}_{1}\right\} \\
\vdots & \vdots \\
\frac{k_{M}^{-1} w}{R T T} & q \in\left\{\mathrm{ss}_{M}, c \mathrm{c}_{M}\right\} \\
0 & \text { otherwise }\end{cases} \\
\phi_{\text {end }}(q, w, s, t): & = \begin{cases}(\text { off }, 0,0) & q \in\left\{\mathrm{ss}_{i}, c \mathrm{ca}_{i}: i=1,2, \ldots, M\right\} \\
(q, w, s) & \text { otherwise. }\end{cases}
\end{aligned}
$$

The intensities and reset maps of the transitions to the congestion avoidance modes must be adapted in the obvious way:

$$
\begin{aligned}
\lambda_{\text {drop }}(q, w, s, t) & := \begin{cases}\frac{p_{\mathrm{drop}}(t) w}{R T T} & q \in\left\{\mathrm{ss}_{i}, c \mathrm{ca}_{i}: i=1,2, \ldots, M\right\} \\
0 & \text { otherwise }\end{cases} \\
\phi_{\mathrm{drop}}(q, w, s, t):= & \begin{cases}\left(c \mathrm{a}_{1}, \frac{w}{2}, s\right) & q=\in\left\{\mathrm{ss}_{1}, c \mathrm{ca}_{1}\right\} \\
\vdots & \vdots \\
\left(c \mathrm{a}_{M}, \frac{w}{2}, s\right) & q=\in\left\{\mathrm{ss}_{M}, c \mathrm{ca}_{M}\right\} \\
(q, w, s) & \text { otherwise }\end{cases}
\end{aligned}
$$

The exponential-sizes model in Sect. 4 is a special case of this for $M=1$. A similar technique could be used to obtain a mixture of exponentials for the distribution of the off periods.

\subsection{Analysis of the TCP SHS models}

To investigate the dynamics of the moments of the sending rate $\mathbf{r}(t)=\frac{\mathbf{w}(t)}{R T T(t)}$ for the mixed-exponentials TCP model, $\forall n \geq 0, q_{0} \in \mathcal{Q}$ we define

$$
\mu_{q_{0}, n}(t):=\mathrm{E}\left[\psi_{q_{0}, n}(\mathbf{q}(t), \mathbf{w}(t), t)\right], \quad \psi_{q_{0}, n}(q, w, t):= \begin{cases}\frac{w^{n}}{R T T(t)^{n}} & q=q_{0} \\ 0 & \text { otherwise. }\end{cases}
$$

From these definitions we conclude that

$$
\mathrm{E}\left[\mathbf{r}^{n}(t)\right]=\sum_{q \in \mathcal{Q}} \mu_{q, n}(t), \quad \forall t \geq 0
$$


and that

$$
\sum_{q \in \mathcal{Q}} \mu_{q, 0}(t)=1, \quad \quad \mu_{\text {off }, n}=0, \quad \forall n \geq 1, t \geq 0 .
$$

The following result can be obtained by directly applying Theorem 1 to the SHS TCP model. Details of the computations can be found in the Appendix.

Theorem 3 (Full-order models). For the mixed-exponentials model in Sect. 4 we have ${ }^{4}$

$$
\begin{aligned}
\dot{\mu}_{\mathrm{off}, 0} & =-\frac{\mu_{\mathrm{off}, 0}}{\tau_{\mathrm{off}}}+\sum_{j=1}^{M} k_{j}^{-1}\left(\mu_{\mathrm{ss}_{j}, 1}+\mu_{\mathrm{ca}_{j}, 1}\right) \\
\dot{\mu}_{\mathrm{ss}_{i}, n} & =\frac{p_{i} w_{0}^{n} \mu_{\mathrm{off}, 0}}{\tau_{\mathrm{off}} R T T^{n}}+n \frac{(\log 2)-n_{\mathrm{ack}} R \dot{T} T}{n_{\mathrm{ack}} R T T} \mu_{\mathrm{ss}_{i}, n}-\left(p_{\mathrm{drop}}+k_{i}^{-1}\right) \mu_{\mathrm{ss}_{i}, n+1} \\
\dot{\mu}_{\mathrm{ca}_{i}, n} & =\frac{n \mu_{\mathrm{ca}_{i}, n-1}}{n_{\mathrm{ack}} R T T^{2}}-\frac{n R \dot{T} T \mu_{\mathrm{ca}_{i}, n}}{R T T}-\left(p_{\mathrm{drop}}+k_{i}^{-1}\right) \mu_{\mathrm{ca}_{i}, n+1}+\frac{p_{\mathrm{drop}}}{2^{n}}\left(\mu_{\mathrm{ss}_{i}, n+1}+\mu_{\mathrm{ca}_{i}, n+1}\right) .
\end{aligned}
$$

\subsection{Reduced-order model}

The system of infinitely many differential equations that appear in Theorem 3 describes exactly the evolution of the moments of the sending rate $\mathbf{r}$ but finding the exact solution to these equations does not appear to be simple. However, as noted by Bohacek [7], Monte Carlo simulations reveal that the steady-state distribution of the sending rate is often well approximated by a Log-Normal distribution. Assuming that on each mode the sending rate $\mathbf{r}$ approximately obeys a Log-Normal distribution even during transients, we can truncate the systems of infinitely many differential equations that appear in Theorem 3 . We recall that, if the random variable $\mathbf{x}$ has a Log-Normal distribution with parameters $\mu$ and $\sigma$, then

$$
\mathrm{E}[\mathbf{x}]=e^{\mu+\frac{\sigma^{2}}{2}}, \quad \mathrm{E}\left[\mathbf{x}^{2}\right]=e^{2 \mu+2 \sigma^{2}}, \quad \mathrm{E}\left[\mathbf{x}^{3}\right]=e^{3 \mu+\frac{9 \sigma^{2}}{2}},
$$

and therefore $\mathrm{E}\left[\mathbf{x}^{3}\right]=\frac{\mathrm{E}\left[\mathbf{x}^{2}\right]^{3}}{\mathrm{E}[\mathbf{x}]^{3}}$. Therefore if $\mathbf{r}$ is approximately Log-Normal distributed in the mode $q \in \mathcal{Q}$, we have that

$$
\mu_{q, 3}=\mu_{q, 0} \mathrm{E}\left[\mathbf{r}^{3} \mid \mathbf{q}=q\right] \approx \mu_{q, 0} \frac{\mathrm{E}\left[\mathbf{r}^{2} \mid \mathbf{q}=q\right]^{3}}{\mathrm{E}[\mathbf{r} \mid \mathbf{q}=q]^{3}}=\frac{\mu_{q, 0} \mu_{q, 2}^{3}}{\mu_{q, 1}^{3}}
$$

where we used the fact that

$$
\mu_{q, n}=\mathrm{P}(\mathbf{q}=q) \mathrm{E}\left[\mathbf{r}^{n} \mid \mathbf{q}=q\right]=\mu_{q, 0} \mathrm{E}\left[\mathbf{r}^{n} \mid \mathbf{q}=q\right] .
$$

Using (24) in (21)-(23), we can eliminate any terms $\mu_{q_{0}, n}, n \geq 3$ in the equations for $\dot{\mu}_{q_{0}, n}, n \leq 2$, thus constructing the following finite-dimensional model to approximately describe the dynamics of the first two moments of the sending rate:

$$
\begin{aligned}
& \dot{\mu}_{\mathrm{ss}_{i}, 0}=\frac{p_{i}\left(1-\sum_{j=1}^{M}\left(\mu_{\mathrm{ss}_{i}, 0}+\mu_{\mathrm{ca}_{i}, 0}\right)\right)}{\tau_{\mathrm{off}}}-\left(p_{\mathrm{drop}}+k_{i}^{-1}\right) \mu_{\mathrm{ss}_{i}, 1} \\
& \dot{\mu}_{\mathrm{ca}_{i}, 0}=p_{\mathrm{drop}} \mu_{\mathrm{ss}_{i}, 1}-k_{i}^{-1} \mu_{\mathrm{ca}_{i}, 1} \\
& \dot{\mu}_{\mathrm{ss}_{i}, 1}=\frac{w_{0} p_{i}\left(1-\sum_{j=1}^{M}\left(\mu_{\mathrm{ss}_{i}, 0}+\mu_{\mathrm{ca}_{i}, 0}\right)\right)}{\tau_{\mathrm{off}} R T T}+\frac{(\log 2)-n_{\mathrm{ack}} R \dot{T} T}{n_{\mathrm{ack}} R T T} \mu_{\mathrm{ss}_{i}, 1}-\left(p_{\mathrm{drop}}+k_{i}^{-1}\right) \mu_{\mathrm{ss}_{i}, 2} \\
& \dot{\mu}_{\mathrm{ca}_{i}, 1}=\frac{\mu_{\mathrm{ca}_{i}, 0}}{n_{\mathrm{ack}} R T T^{2}}-\frac{R T T \mu_{\mathrm{ca}_{i}, 1}}{R T T}+\frac{p_{\mathrm{drop}} \mu_{\mathrm{ss}_{i}, 2}}{2}-\left(\frac{p_{\mathrm{drop}}}{2}+k_{i}^{-1}\right) \mu_{\mathrm{ca}_{i}, 2} \\
& \dot{\mu}_{\mathrm{ss}_{i}, 2}=\frac{w_{0}^{2} p_{i}\left(1-\sum_{j=1}^{M}\left(\mu_{\mathrm{ss}_{i}, 0}+\mu_{\mathrm{ca}_{i}, 0}\right)\right)}{\tau_{\mathrm{off}} R T T^{2}}+\frac{(\log 4) \mu_{\mathrm{ss}_{i}, 2}}{n_{\mathrm{ack}} R T T}-\frac{2 R \dot{T} T \mu_{\mathrm{ss}_{i}, 2}}{R T T}-\left(p_{\mathrm{drop}}+k_{i}^{-1}\right) \frac{\mu_{\mathrm{ss}_{i}, 0} \mu_{\mathrm{ss}_{i}, 2}^{3}}{\mu_{\mathrm{ss}_{i}, 1}^{3}}
\end{aligned}
$$

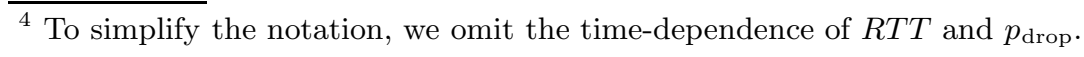




$$
\dot{\mu}_{c a_{i}, 2}=\frac{2 \mu_{c a_{i}, 1}}{n_{\mathrm{ack}} R T T^{2}}-\frac{2 R \dot{T} T \mu_{c a_{i}, 2}}{R T T}+\frac{p_{\mathrm{drop}}}{4} \frac{\mu_{\mathrm{ss}_{i}, 0} \mu_{\mathrm{ss}_{i}, 2}^{3}}{\mu_{\mathrm{ss}_{i}, 1}^{3}}-\left(\frac{3 p_{\mathrm{drop}}}{4}+k_{i}^{-1}\right) \frac{\mu_{\mathrm{ca}_{i}, 0} \mu_{c a_{i}, 2}^{3}}{\mu_{\mathrm{ca}_{i}, 1}^{3}} .
$$

We present next simulations of this reduced model for a few representative parameter values.

Figure 4 corresponds to a transfer-size distribution that results from the mixture of two exponentials $(M=2)$ with parameters

$$
p_{1}=88.87 \%, \quad k_{1}=3.5 \mathrm{~KB}, \quad p_{2}=11.13 \%, \quad k_{2}=246 \mathrm{~KB} .
$$

The first exponential corresponds to small "mice" transfers (3.5KB average) and the second to "elephant" mid-size transfers (246KB average). The small transfers are assumed more common (88.87\%). These parameters result in a distribution with an average transfer-size of $30.58 \mathrm{~KB}$ and for which $11.13 \%$ of the transfers account for $89.7 \%$ of the total volume transfered. This is consistent with the file distribution observed in the UNIX file system [21]. However, it does not accurately capture the tail of the distribution (it lacks the "mammoth" files that will be considered later). The results obtained with the reduced model (25)-(30) match reasonably well those obtained from Monte Carlo simulations of the full SHS model, especially taking into account the very large standard deviations. It is worth it to point out that the simulation of (25)-(30) takes just a few seconds, whereas each Monte Carlo simulation takes several hours of CPU.

Two somewhat surprising conclusions can be drawn from Figure 4 for this distribution of transfer-sizes and off-times:

1. The average total sending rate varies very little with the drop rate (at least up to the drop rate of $33 \%$ shown in the plots), with most of the packets transmitted belonging to "elephant" mid-size transfers. This is perhaps not surprising when most packets are transmitted in the slow-start mode (for drop rates below .8\%) but still holds when a significant fraction of packets are sent in the congestion avoidance mode.

2. The dynamics of TCP are completely dominated by second order moments. In Figure 4, the standard deviation is 5 to 20 times larger than the average sending rate, which is very accurately predicted by the reduced model. As the drop rate increases, the standard deviation decreases but even for $p_{\text {drop }}=33 \%$ the standard deviation is still 5 times larger than the average sending rate.

This behavior is completely different from the one observed for TCP flows that are always on, for which it has been shown that the steady-state average sending rate is approximately given by $\frac{c}{R T T \sqrt{p_{\text {drop }}}}$, where $R T T$ denotes the average round-trip time, $p_{\text {drop }}$ the per-packet drop rate, and $c$ a constant ranging from 1.225 to 1.310 depending on the method used to derive the equation $[8,19,32,33,37]$. This equation is valid at least for simple network topologies, small values of $p_{\text {drop }}$, and one acknowledgment per ACK packet $\left(n_{\text {ack }}=1\right)$. Generalizations can be found, e.g., in [38, 42].

We considered next a transfer-size distribution that results from a mixture of three exponentials $(M=3)$ with parameters

$$
\begin{aligned}
& p_{1}=98 \%, \quad p_{2}=1.7 \%, \quad p_{3}=.02 \%, \\
& k_{1}=6 \mathrm{~KB}, \quad k_{2}=400 \mathrm{~KB}, \quad k_{3}=10 \mathrm{MB} .
\end{aligned}
$$

The first exponential corresponds to small "mice" transfers, the second to mid-size "elephant" transfers, and the third to large "mammoth" transfers. The resulting distribution, shown in Figure 5, approximates the one reported by Arlitt et al. [2] obtained from monitoring transfers from a world-wide web proxy within an Internet Service Provider. This distribution has a much heavier tail than the one considered before.

Figure 6 contains results obtained from the reduced model. We do not present Monte Carlo results because the simulation times needed to capture the tails of the transfer-size distribution are prohibitively large. In this figure we varied the average off-time $\tau_{\text {off }}$ from .2 to 5 seconds. This essentially scales the sending rate but does not significantly change the way it varies with the drop probability. It turns out that the main 

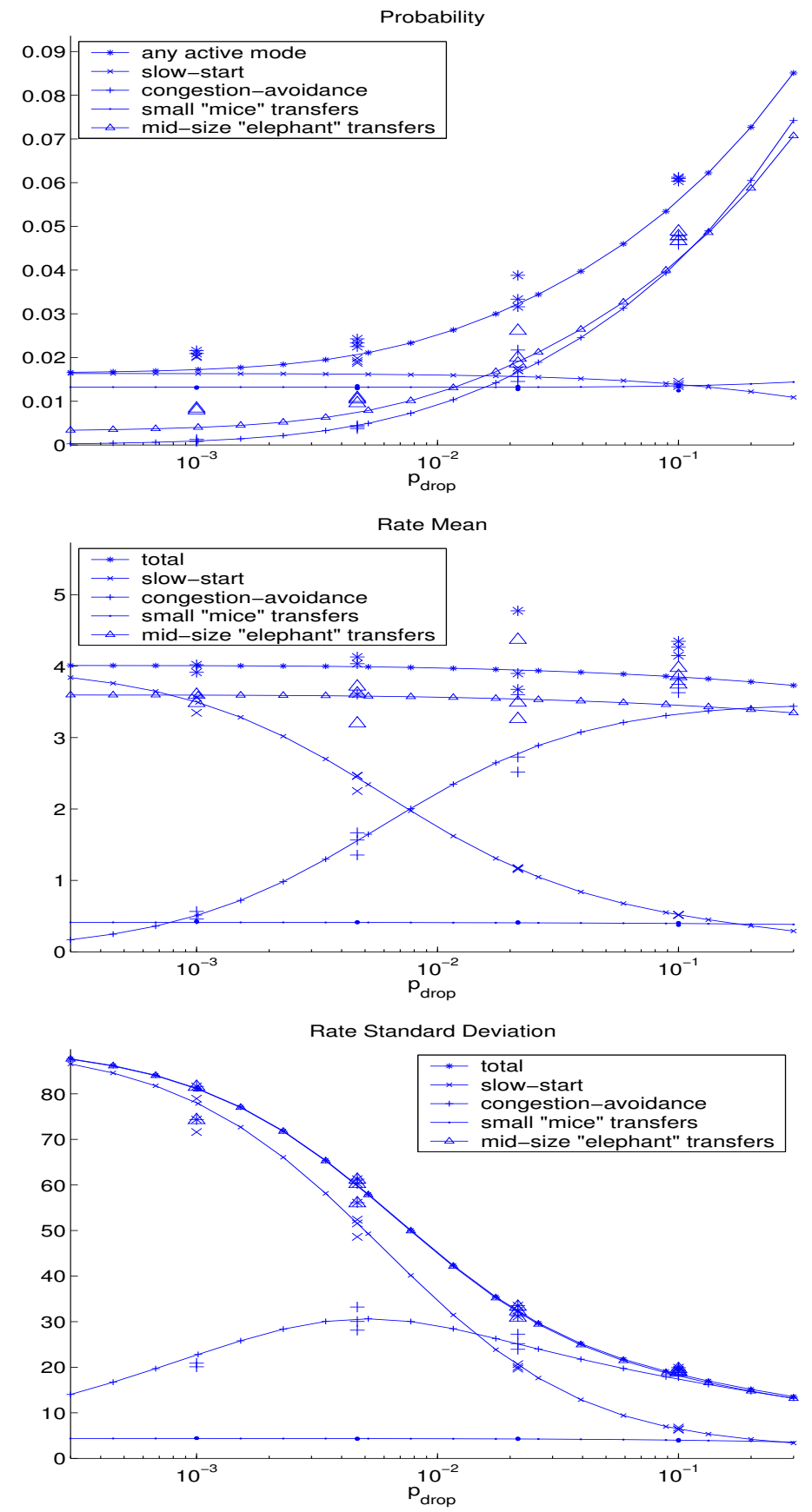

Fig. 4. Steady-state values for the probability of a flow being on each mode (top) and the average (mid) and standard deviation (bottom) of the sending rate as a function of the drop probability. The solid lines were obtained for the model (25)-(30) with $R T T=50 \mathrm{~ms}, n_{\text {ack }}=1$, and a transfer-size distribution that results from the mixture of two exponentials with the parameters in (31). The mean off time was set to $\tau_{\text {off }}=5$ sec. The (larger) symbols were obtained from Monte Carlo Simulations. 


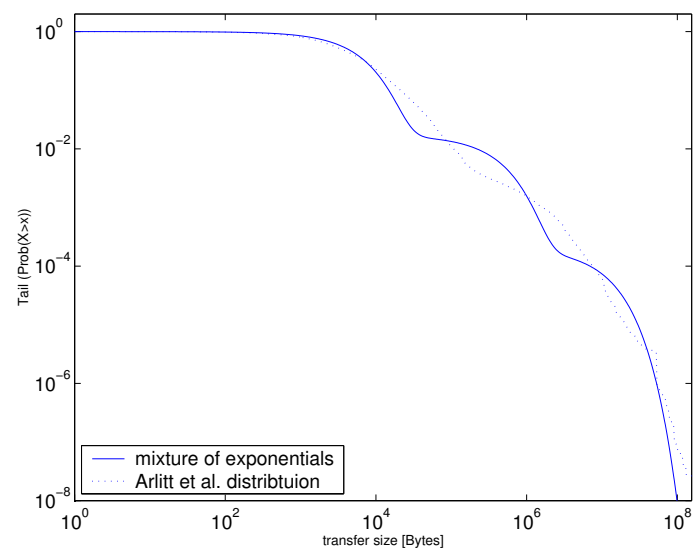

Fig. 5. Distribution of transfer-sizes resulting from the mixture of three exponentials with the parameters in (32), which were used in the simulations in Figure 6.

conclusions drawn before still hold: the average sending rate varies relatively little with the drop rate and the dynamics of TCP are completely dominated by second order moments. The mid-size "elephants" still dominate followed by the small "mice." The large "mammoth" transfers occur at a rate that is not sufficiently large to have a significant impact on the average sending rate.

\section{Conclusions}

This paper presents a new model for SHSs where transitions between discrete modes are triggered by stochastic events, which occur at rates that are allowed to depend on both the continuous and the discrete states of the SHS. Based on results available for Piecewise-Deterministic Markov Process (PDPs), we provide a formula for the extended generator of the SHS.

As an illustration, we presented a SHS model for on-off TCP flows that considers both slow-start and congestion avoidance. One important observation that stems from this work is that for realistic transfer-size distributions high-order statistical moments seem to dominate the dynamics of TCP. Also, the probability of drop appears to have a much larger effect on the standard deviation of the sending rate than on its mean value. We are currently investigating the impact of this observation on the stability and performance of congestion control mechanisms.

This work opens several avenues for future research. We would like to determine conditions under which Assumption 2(ii) holds, milder than those required by Lemma 1. It is also worth to investigate how solutions to deterministic hybrid systems that exist only in a finite interval due to Zeno phenomena can be globally extended using SHSs. Finally, one needs to develop general tools to approximate the dynamics of SHSs by finite-dimensional systems of ODEs, as was done for the TCP example.

\section{Acknowledgments}

The author would like to thank Roger Brockett for providing a preprint of [11]; Martin Arlitt for making available the processed data regarding the transfer-size distribution reported in [2]; Sanjoy Mitter and John Lygeros for provided several relevant references; and Stephan Bohacek, Yonggang Xu, and Guillaume Bonnet for insightful comments. 

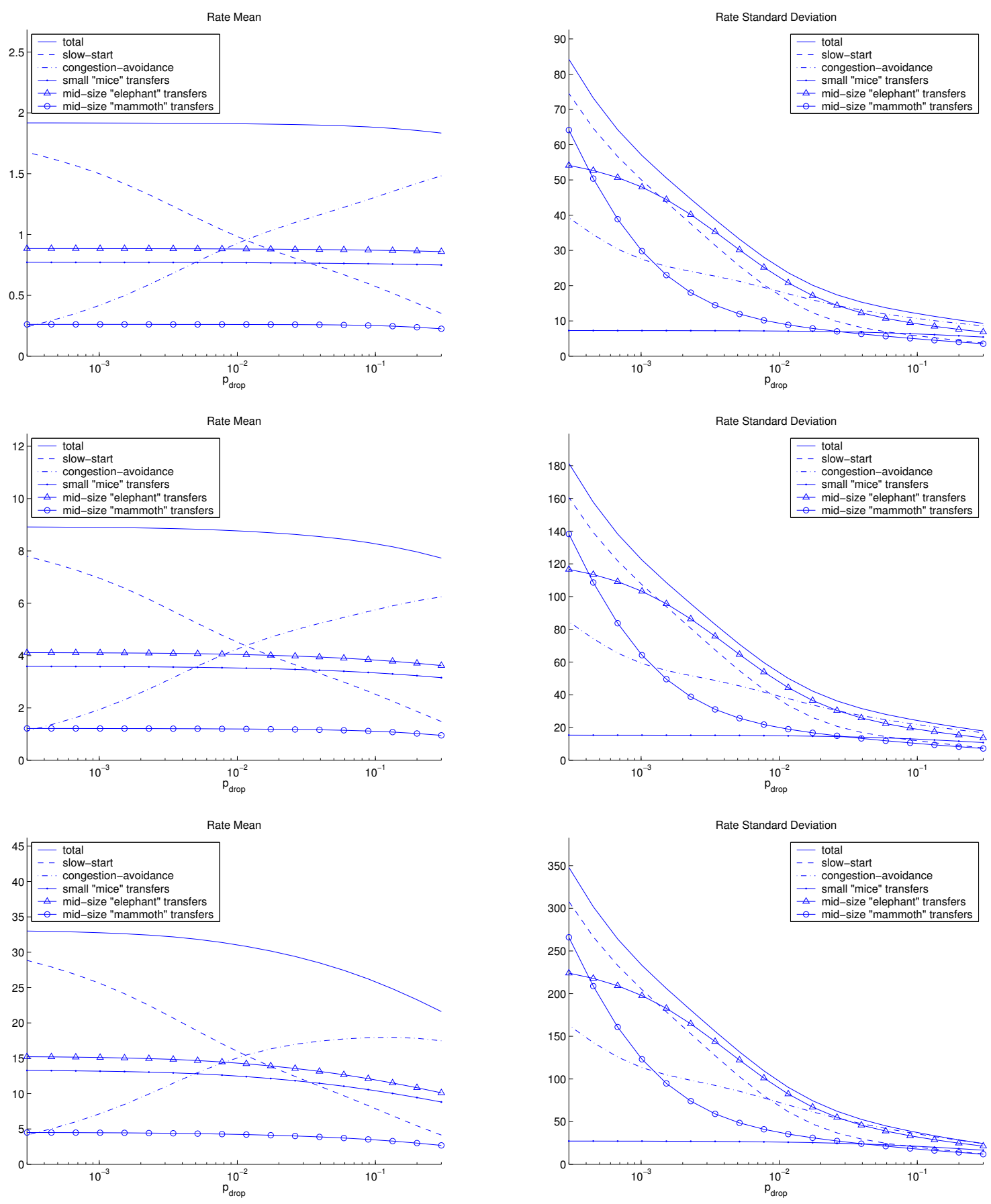

Fig. 6. Steady-state values for the average (left) and standard deviation (right) of the sending rate as a function of the drop probability. These results were obtained from the reduced model, with $R T T=50 \mathrm{~ms}, n_{\text {ack }}=1$, and a transfer-size distribution resulting from the mixture of three exponentials with the parameters in (32). The mean off time was set to $\tau_{\text {off }}=5 \mathrm{sec}$ (top), 1sec (mid), and 0.2sec (bottom). 


\section{Appendix}

Proof of Equation (5). Consider an interval $(t, t+d t], d t>0$ and let $\mathbf{t}_{k}$ be the largest transition time smaller than or equal to $t$. The probability that the counter $\mathbf{N}_{\ell}$ will be incremented in $(t, t+d t]$ is given by

$$
\begin{aligned}
\mathrm{P}\left(\mathbf{N}_{\ell}(t+d t)>\mathbf{N}_{\ell}(t)\right)=\mathrm{P}\left(e^{-\int_{\mathbf{t}_{k}}^{t+d t} \boldsymbol{\lambda}(s) d s} \leq \boldsymbol{\mu}_{k}^{\ell}<e^{-\int_{\mathbf{t}_{k}}^{t} \boldsymbol{\lambda}(s) d s}\right) & = \\
= & \mathrm{E}\left[\mathrm{P}\left(e^{-\int_{\mathbf{t}_{k}}^{t+d t} \boldsymbol{\lambda}(s) d s} \leq \boldsymbol{\mu}_{k}^{\ell}<e^{-\int_{\mathbf{t}_{k}}^{t} \boldsymbol{\lambda}(s) d s} \mid \mathbf{t}_{k}, \mathbf{q}\left(\mathbf{t}_{k}\right), \mathbf{x}\left(\mathbf{t}_{k}\right)\right)\right],
\end{aligned}
$$

where $\boldsymbol{\lambda}(s):=\lambda_{\ell}\left(\mathbf{q}\left(\mathbf{t}_{k}\right), \varphi\left(s ; \mathbf{t}_{k}, \mathbf{q}\left(\mathbf{t}_{k}\right), \mathbf{x}\left(\mathbf{t}_{k}\right)\right), s\right)$ and the expectation is taken with respect to the random variables $\mathbf{t}_{k}, \mathbf{q}\left(\mathbf{t}_{k}\right), \mathbf{x}\left(\mathbf{t}_{k}\right)$. Since $\boldsymbol{\mu}_{k}^{\ell}$ is uniformly distributed in $[0,1]$, we have that

$$
\mathrm{P}\left(e^{-\int_{\mathbf{t}_{k}}^{t+d t} \boldsymbol{\lambda}(s) d s} \leq \boldsymbol{\mu}_{k}^{\ell}<e^{-\int_{\mathbf{t}_{k}}^{t} \boldsymbol{\lambda}(s) d s} \mid \mathbf{t}_{k}, \mathbf{q}\left(\mathbf{t}_{k}\right), \mathbf{x}\left(\mathbf{t}_{k}\right)\right)=e^{-\int_{\mathbf{t}_{k}}^{t} \boldsymbol{\lambda}(s) d s}-e^{-\int_{\mathbf{t}_{k}}^{t+d t} \boldsymbol{\lambda}(s) d s}
$$

with probability one, and therefore

$$
\mathrm{P}\left(\mathbf{N}_{\ell}(t+d t)>\mathbf{N}_{\ell}(t)\right)=\mathrm{E}\left[e^{-\int_{\mathbf{t}_{k}}^{t} \lambda_{\ell}(\mathbf{q}(t), \varphi(s ; t, \mathbf{q}(t), \mathbf{x}(t)), s) d s}-e^{-\int_{\mathbf{t}_{k}}^{t+d t} \lambda_{\ell}(\mathbf{q}(t), \varphi(s ; t, \mathbf{q}(t), \mathbf{x}(t)), s) d s}\right],
$$

where we used the facts that $\varphi\left(s ; \mathbf{t}_{k}, \mathbf{q}\left(\mathbf{t}_{k}\right), \mathbf{x}\left(\mathbf{t}_{k}\right)\right)=\varphi(s ; t, \mathbf{q}(t), \mathbf{x}(t))$ and that $\mathbf{q}\left(\mathbf{t}_{k}\right)=\mathbf{q}(t)$ with probability one because there are no resets in $\left(\mathbf{t}_{k}, t\right]$ due to the definition of $\mathbf{t}_{k}$. Equation (5) follows from dividing both sides of (33) by $d t$ and taking the limit as $d t \downarrow 0$.

Proof of Lemma 1. Let $(q, x)$ denote a sample path of $(\mathbf{q}, \mathbf{x})$ constructed according to the algorithm in Sect. 2.1 with initial conditions $\left(q_{0}, x_{0}, t_{0}\right) \in \mathcal{Q} \times \mathbb{R}^{n} \times[0, \infty)$ and $t_{i}, i \geq 0$ the corresponding transition times. On each interval $\left[t_{i}, t_{i+1}\right), x$ evolves according to $(1)$ and therefore

$$
x(s)=x\left(t_{i}\right)+\int_{t_{i}}^{s} f\left(q\left(t_{i}\right), x(r), r\right) d r, \quad \forall s \in\left[t_{i}, t_{i+1}\right) .
$$

Taking norms we obtain

$$
\|x(s)\| \leq\left\|x\left(t_{i}\right)\right\|+\int_{t_{i}}^{s} \max \left\{\gamma_{f}(r)\|x(r)\|, c_{f}\right\} d r, \quad \forall s \in\left[t_{i}, t_{i+1}\right) .
$$

Since $\gamma_{f}(r)\|x(r)\|$ is continuous on $\left[t_{i}, t_{i+1}\right)$, one of the following three cases must occur:

1. $\gamma_{f}(r)\|x(r)\|>c_{f}, \forall r \in\left[t_{i}, t_{i+1}\right)$. In this case,

$$
\|x(s)\| \leq\left\|x\left(t_{i}\right)\right\|+\int_{t_{i}}^{s} \gamma_{f}(r)\|x(r)\| d r, \quad \forall s \in\left[t_{i}, t_{i+1}\right),
$$

and we conclude using the Bellman-Gronwall Lemma that

$$
\|x(s)\| \leq e^{\int_{t_{i}}^{s} \gamma_{f}(r) d r}\left\|x\left(t_{i}\right)\right\|, \quad \forall s \in\left[t_{i}, t_{i+1}\right) .
$$

2. $\gamma_{f}\left(r^{*}\right)\left\|x\left(r^{*}\right)\right\|=c_{f}$ for some $r^{*} \in\left[t_{i}, t_{i+1}\right)$ and $\gamma_{f}(r)\|x(r)\| \geq c_{f}, \forall r \in\left[r^{*}, t_{i+1}\right)$. In this case, we conclude from applying the Bellman-Gronwall Lemma on $\left[r^{*}, t_{i+1}\right)$ that

$$
\|x(s)\| \leq e^{\int_{r^{*}}^{s} \gamma_{f}(r) d r}\left\|x\left(r^{*}\right)\right\|=\frac{e^{\int_{r^{*}}^{s} \gamma_{f}(r) d r} c_{f}}{\gamma_{f}\left(r^{*}\right)} \quad \forall s \in\left[r^{*}, t_{i+1}\right) .
$$

3. $\gamma_{f}(r)\|x(r)\|<c_{f}, \forall r \in\left[t_{i}, t_{i+1}\right)$. 
Without loss of generality we assume that the function $\gamma_{f}$ is monotone increasing and $\gamma_{f}\left(t_{0}\right)>0$. For any of the cases above, it is then always true that

$$
\|x(s)\| \leq e^{\int_{t_{i}}^{s} \gamma_{f}(r) d r} \max \left\{\left\|x\left(t_{i}\right)\right\|, \frac{c_{f}}{\gamma_{f}\left(t_{0}\right)}\right\}, \quad \forall s \in\left[t_{i}, t_{i+1}\right)
$$

and assuming that the discontinuity at time $t_{i+1}$ is due to the transition $\phi_{\ell}$, we have that

$$
\left\|x\left(t_{i+1}\right)\right\|=\left\|\phi_{\ell}^{x}\left(q\left(t_{i}\right), x^{-}\left(t_{i+1}\right), t_{i+1}\right)\right\| \leq \max \left\{e^{\int_{t_{i}}^{t_{i+1}} \gamma_{f}(r) d r}\left\|x\left(t_{i}\right)\right\|, \frac{e^{\int_{t_{i}}^{t_{i+1}} \gamma_{f}(r) d r} c_{f}}{\gamma_{f}\left(t_{0}\right)}, c_{\phi}\right\} .
$$

In the worst case, $\left\|x_{0}\right\|$ is larger than both $\frac{c_{f}}{\gamma_{f}\left(t_{0}\right)}$ and $c_{\phi}$ and the first term always dominates as one iterates from $i=0$ to $i=k-1$, which yields

$$
\left\|x\left(t_{k}\right)\right\| \leq e^{\int_{t_{0}}^{t_{k}} \gamma_{f}(r) d r} \max \left\{\left\|x_{0}\right\|, \frac{c_{f}}{\gamma_{f}\left(t_{0}\right)}, c_{\phi}\right\} .
$$

Take know an arbitrary time $t \geq 0$ and let $t_{k}$, denote the last transition time before or at $t$. From (35) and (34) for $i=k$ and $s=t$, we conclude that

$$
\|x(t)\| \leq e^{\int_{t_{0}}^{t} \gamma_{f}(r) d r} \max \left\{\left\|x_{0}\right\|, \frac{c_{f}}{\gamma_{f}\left(t_{0}\right)}, c_{\phi}\right\}
$$

which finishes the proof.

Proof of Equation (14). For a particular $\epsilon>0$, the extended generator of the SHS is given by

$$
\begin{aligned}
(L \psi)(q, x, t)=\frac{\partial \psi(q, x, t)}{\partial x} f(q, x, t) & +\frac{\partial \psi(q, x, t)}{\partial t}+\sum_{\ell=1}^{m}\left(\psi\left(\phi_{\ell}(q, x, t), t\right)-\psi(q, x, t)\right) \lambda_{\ell}(q, x, t)+ \\
& +\sum_{i=1}^{k} \frac{\psi\left(q, x+\sqrt{\epsilon} g_{i}(q, x, t), t\right)+\psi\left(q, x-\sqrt{\epsilon} g_{i}(q, x, t), t\right)-2 \psi(q, x, t)}{2 \epsilon}
\end{aligned}
$$

To take the limit as $\epsilon \downarrow 0$, we compute the Taylor series around $\epsilon=0$ of the numerator of each term in the last summation, which yields

$$
\begin{aligned}
\psi\left(q, x+\sqrt{\epsilon} g_{i}(q, x, t), t\right)+\psi\left(q, x-\sqrt{\epsilon} g_{i}(q, x, t), t\right)-2 \psi(q, x, t) & \\
& =\epsilon g_{i}(q, x, t)^{\prime} \frac{\partial^{2} \psi(q, x, t)}{\partial x^{2}} g_{i}(q, x, t)+O\left(\epsilon^{\frac{3}{2}}\right)
\end{aligned}
$$

where $O\left(\epsilon^{\frac{3}{2}}\right)$ a term of order no larger than $\epsilon^{\frac{3}{2}}$. Equation (14) is then obtained by making $\epsilon \downarrow 0$.

Verification of (19). To verify that (19) is consistent with a distribution $F_{\mathbf{s}^{*}}:[0, \infty) \rightarrow[0,1]$ for the number $\mathbf{s}^{*}$ of packets sent in each TCP session, consider a TCP session that started at some transition time $\mathbf{t}_{k}$ and transmits with sending rate $\mathbf{r}$. The probability that the number $\mathbf{s}^{*}$ of packets sent in this session is larger than $s>0$ is given by

$$
\mathrm{P}\left(\mathbf{s}^{*} \geq s \mid \mathbf{r}, \mathbf{t}_{k}\right)=\mathrm{P}\left(\mathbf{N}_{\mathrm{ses}}\left(\mathbf{t}_{k}\right)=\mathbf{N}_{\mathrm{ses}}(\mathbf{t}) \mid \mathbf{r}, \mathbf{t}_{k}\right)
$$

where $\mathbf{t}>0$ is defined such that $\int_{\mathbf{t}_{k}}^{\mathbf{t}} \mathbf{r}(\tau) d \tau=s$. Denoting by $\mathbf{t}_{k+m}$ the last transition time before $\mathbf{t}$, we have that

$$
\mathrm{P}\left(\mathbf{s}^{*} \geq s \mid \mathbf{r}, \mathbf{t}_{k}\right)=\mathrm{P}\left(e^{-\int_{\mathbf{t}_{k+m}}^{\mathbf{t}} \frac{\mathbf{r}(\tau) F_{\mathbf{s}^{*}}^{\prime}(\mathbf{s}(\tau))}{1-F_{\mathbf{s}^{*}}(\mathbf{s}(\tau))} d \tau}>\boldsymbol{\mu}_{k+m}^{\mathrm{ses}}, e^{-\int_{\mathbf{t}_{i}}^{\mathbf{t}_{i+1}} \frac{\mathbf{r}(\tau) F_{\mathbf{s}^{*}}^{\prime}(\mathbf{s}(\tau))}{1-F_{\mathbf{s}^{*}}(\mathbf{s}(\tau))} d \tau}>\boldsymbol{\mu}_{i}^{\mathrm{ses}}, \forall k \leq i \leq k+m \mid \mathbf{r}, \mathbf{t}_{k}\right)
$$




$$
=\mathrm{P}\left(\frac{1-F_{\mathbf{s}^{*}}(\mathbf{s}(\mathbf{t}))}{1-F_{\mathbf{s}^{*}}\left(\mathbf{s}\left(\mathbf{t}_{k+m}\right)\right)}>\boldsymbol{\mu}_{k+m}^{\mathrm{ses}}, \frac{1-F_{\mathbf{s}^{*}}\left(\mathbf{s}\left(\mathbf{t}_{i+1}\right)\right)}{1-F_{\mathbf{s}^{*}}\left(\mathbf{s}\left(\mathbf{t}_{i}\right)\right)}>\boldsymbol{\mu}_{i}^{\mathrm{ses}}, \forall k \leq i \leq k+m \mid \mathbf{r}, \mathbf{t}_{k}\right),
$$

where we used the fact that

$$
\frac{\partial}{\partial \tau} \log \left(1-F_{\mathbf{s}^{*}}(\mathbf{s}(\tau))\right)=-\frac{\mathbf{r}(\tau) F_{\mathbf{s}^{*}}^{\prime}(\mathbf{s}(\tau))}{1-F_{\mathbf{s}^{*}}(\mathbf{s}(\tau))} . \quad \forall \tau \geq 0 .
$$

Since all the events in the right-hand-side of (36) are conditionally independent given $\mathbf{r}, \mathbf{t}_{k}$ and the random variables $\boldsymbol{\mu}_{i}^{\mathrm{ses}}$ are uniformly distributed in $[0,1]$, we conclude that

$$
\begin{aligned}
\mathrm{P}\left(\mathbf{s}^{*} \geq s \mid \mathbf{r}, \mathbf{t}_{k}\right) & =\mathrm{P}\left(\frac{1-F_{\mathbf{s}^{*}}(\mathbf{s}(\mathbf{t}))}{1-F_{\mathbf{s}^{*}}\left(\mathbf{s}\left(\mathbf{t}_{k+m}\right)\right)}>\boldsymbol{\mu}_{k+m}^{\mathrm{ses}} \mid \mathbf{r}, \mathbf{t}_{k}\right) \prod_{i=k}^{k+m} \mathrm{P}\left(\frac{1-F_{\mathbf{s}^{*}}\left(\mathbf{s}\left(\mathbf{t}_{i+1}\right)\right)}{1-F_{\mathbf{s}^{*}}\left(\mathbf{s}\left(\mathbf{t}_{i}\right)\right)}>\boldsymbol{\mu}_{i}^{\mathrm{ses}} \mid \mathbf{r}, \mathbf{t}_{k}\right), \\
& =1-F_{\mathbf{s}^{*}}\left(\int_{\mathbf{t}_{k}}^{\mathbf{t}} \mathbf{r}(\tau) d \tau\right)=1-F_{\mathbf{s}^{*}}(s),
\end{aligned}
$$

which confirms that $F_{\mathbf{S}^{*}}$ is indeed the cumulative distribution of the number of packets sent per TCP session.

Proof of Theorem 3. Applying the extended generator $L$ for the SHS model defined in Sect. 4 to the functions $\psi_{q_{0}, n}$ defined in (20) yields

$$
\begin{aligned}
\left(L \psi_{q_{0}, n}\right)(q, w, s, t)= & \frac{\partial \psi_{q_{0}, n}(q, w, t)}{\partial w} f(q, w, s, t)+\frac{\partial \psi_{q_{0}, n}(q, w, t)}{\partial t} \\
& +\left(\psi_{q_{0}, n}\left(\phi_{\mathrm{drop}}(q, w, s, t), t\right)-\psi_{q_{0}, n}(q, w, t)\right) \lambda_{\mathrm{drop}}(q, w, s, t) \\
& +\left(\psi_{q_{0}, n}\left(\phi_{\mathrm{end}}(q, w, s, t), t\right)-\psi_{q_{0}, n}(q, w, t)\right) \lambda_{\mathrm{end}}(q, w, s, t) \\
& +\sum_{j=1}^{M}\left(\psi_{q_{0}, n}\left(\phi_{j}(q, w, s, t), t\right)-\psi_{q_{0}, n}(q, w, t)\right) \lambda_{j}(q, w, s, t),
\end{aligned}
$$

from which we obtain by direct computation that

$$
\begin{aligned}
& \left(L \psi_{\text {off.o }}\right)(q, w, t)= \begin{cases}\frac{k_{1}^{-1} w}{R T T} & q \in\left\{\mathrm{ss}_{1}, c \mathrm{a}_{1}\right\} \\
\vdots & \vdots \\
\frac{k_{M}^{-1} w}{R T T} & q \in\left\{\mathrm{ss}_{M}, c \mathrm{a}_{M}\right\} \\
-\frac{1}{\tau_{\text {off }}} & q=\text { off } \\
0 & \text { otherwise }\end{cases} \\
& =-\frac{\psi_{\mathrm{off}, 0}(q, w, t)}{\tau_{\mathrm{off}}}+\sum_{j=1}^{M} k_{j}^{-1}\left(\psi_{\mathrm{ss}_{j}, 1}(q, w, t)+\psi_{\mathrm{ca}_{j}, 1}(q, w, t)\right) \\
& \left(L \psi_{\text {ss }_{i}, n}\right)(q, w, t)= \begin{cases}\frac{n\left((\log 2) n_{\text {ack }}^{-1}-R \dot{T} T\right) w^{n}-\left(p_{\mathrm{drop}}+k_{i}^{-1}\right) w^{n+1}}{R T T^{n+1}} & q=s S_{i} \\
\frac{p_{i} w_{0}^{n}}{\tau_{\mathrm{off}} R T T^{n}} & q=\text { off } \\
0 & \text { otherwise }\end{cases} \\
& =\frac{p_{i} w_{0}^{n} \psi_{\text {off }, 0}(q, w, t)}{\tau_{\text {off }} R T T^{n}}+n \frac{(\log 2)-n_{\text {ack }} R \dot{T} T}{n_{\text {ack }} R T T} \psi_{\text {ss }_{i}, n}(q, w, t)-\left(p_{\text {drop }}+k_{i}^{-1}\right) \psi_{\mathrm{ss}_{i}, n+1}(q, w, t) \\
& \left(L \psi_{\mathrm{ca}_{i}, n}\right)(q, w, t)= \begin{cases}\frac{2^{-n} p_{\mathrm{drop}} w^{n+1}}{R T T^{n+1}} & q=s S_{i} \\
\frac{n n_{\text {ack }}^{-1} w^{n-1}-n R T \dot{T} T w^{n}-\left(p_{\mathrm{drop}}+k_{i}^{-1}-2^{-n} p_{\mathrm{drop}}\right) w^{n+1}}{R T T^{n+1}} & q=c a_{i} \\
0 & \text { otherwise }\end{cases}
\end{aligned}
$$




$$
\begin{gathered}
=\frac{n \psi_{\mathrm{ca}_{i}, n-1}(q, w, t)}{n_{\mathrm{ack}} R T T^{2}}-\frac{n R \dot{T} T \psi_{\mathrm{ca}_{i}, n}(q, w, t)}{R T T}-\left(p_{\mathrm{drop}}+k_{i}^{-1}\right) \psi_{\mathrm{ca}_{i}, n+1}(q, w, t) \\
+\frac{p_{\mathrm{drop}}\left(\psi_{\mathrm{ss}_{i}, n+1}(q, w, t)+\psi_{\mathrm{ca}_{i}, n+1}(q, w, t)\right)}{2^{n}} .
\end{gathered}
$$

To obtain (21)-(23), we use (10) to conclude that

$$
\dot{\mu}_{q_{0}, n}=\mathrm{E}\left[\left(L \psi_{q_{0}, n}\right)(\mathbf{q}, \mathbf{w}, t)\right],
$$

and replace in the right-hand-side of this equation the expectations of the $\psi_{q_{0}, n}$ by the corresponding $\mu_{q_{0}, n}$.

\section{Bibliography}

[1] P. J. Antsaklis, J. A. Stiver, and M. D. Lemmon. Hybrid system modeling and autonomous control systems. In Grossman et al. [18], pages 366-392.

[2] M. Arlitt, R. Friedrich, and T. Jin. Workload characterization of a web proxy in a cable modem environment. Technical Report HPL-1999-48, Hwelett-Packard Laroratories, Palo Alto, CA, Apr. 1999.

[3] A. Back, J. Guckenheimer, and M. Myers. A dynamical simulation facility for hybrid systems. In Grossman et al. [18].

[4] P. Barford, A. Bestavros, A. Bradley, and M. Crovella. Changes in web client access patterns. World Wide Web, Special Issue on Characterization and Performance Evaluation, 2(1-2):15-28, 1999.

[5] K. Benjelloun and E. K. Boukas. Mean square stochastic stability of linear time-delay system with Markovian jumping parameters. IEEE Trans. on Automat. Contr., 43(10):1456-1460, Oct. 1998.

[6] A. Bensoussan and J.-L. Lion. Impulse Control and Quasi-Variational Inequalities. Gauthier-Villars, Paris, 1984. Translation to English of Contrôle impulsionnel et inéquations quasi-variationnelles, 1982.

[7] S. Bohacek. A stochastic model of TCP and fair video transmission. In Proc. of the IEEE INFOCOM, 2003.

[8] S. Bohacek, J. P. Hespanha, J. Lee, and K. Obraczka. Analysis of a TCP hybrid model. In Proc. of the 39th Annual Allerton Conf. on Comm., Contr., and Computing, Oct. 2001.

[9] S. Bohacek, J. P. Hespanha, J. Lee, and K. Obraczka. A hybrid systems modeling framework for fast and accurate simulation of data communication networks. In Proc. of the ACM Int. Conf. on Measurements and Modeling of Computer Systems (SIGMETRICS), June 2003.

[10] M. S. Branicky, V. S. Borkar, and S. K. Mitter. A unified framework for hybrid control: Background, model and theory. In Proc. of the 33rd Conf. on Decision and Contr., volume 4, pages 4228-4234, Dec. 1994.

[11] R. Brockett. Lecture notes on stochastic control. Provided by the author, 2002.

[12] R. W. Brockett. Hybrid models for motion control systems. In H. L. Trentelman and J. C. Willems, editors, Essays in Control: Perspectives in the Theory and its Applications, pages 29-53. Birkhäuser, Boston, 1993.

[13] O. L. V. Costa and M. D. Fragoso. Stability results for discrete-time linear systems with Markovian jumping parameters. J. Mathematical Anal. and Applications, 179:154-178, 1993.

[14] M. H. A. Davis. Markov models and optimization. Monographs on statistics and applied probability. Chapman \& Hall, London, UK, 1993.

[15] Y. Fang and K. A. Loparo. Stabilization of continuous-time jump linear systems. IEEE Trans. on Automat. Contr., 47(10):1590-1603, Oct. 2002.

[16] J. A. Filar, V. Gaitsgory, and A. B. Haurie. Control of singularly perturbed hybrid stochastic systems. IEEE Trans. on Automat. Contr., 46(2):179-190, Feb. 2001.

[17] M. K. Ghosh, A. Arapostathis, and S. Marcus. Ergodic control of switching diffusions. SIAM J. Contr. Optimization, 35(6):1952-1988, Nov. 1997.

[18] R. L. Grossman, A. Nerode, A. P. Ravn, and H. Rishel, editors. Hybrid Systems, volume 736 of Lect. Notes in Comput. Science. Springer-Verlag, New York, 1993. 
[19] J. P. Hespanha, S. Bohacek, K. Obraczka, and J. Lee. Hybrid modeling of TCP congestion control. In M. D. D. Benedetto and A. Sangiovanni-Vincentelli, editors, Hybrid Systems: Computation and Control, number 2034 in Lect. Notes in Comput. Science, pages 291-304. Springer-Verlag, Berlin, Mar. 2001.

[20] J. Hu, J. Lygeros, and S. Sastry. Towards a theory of stochastic hybrid systems. In Lynch and Krogh [31], pages 160-173.

[21] G. Irlam. Unix file size survey - 1993. Available at http://www.base.com/gordoni/ufs93.html, Nov. 1994.

[22] K. Itô. Stochastic integral. Proc. of the Imperial Academy of Tokyo, 20:519-524, 1944.

[23] J. Jacod and A. N. Shiriaev. Limit Theorems for Stochastic Processes. Springer Verlag, 2nd edition, 2002.

[24] K. H. Johansson, M. Egerstedt, J. Lygeros, and S. Sastry. On the regularization of Zeno hybrid automata. Syst. $\&$ Contr. Lett., 38:141-150, 1999.

[25] S. Kunniyur and R. Srikant. Analysis and design of an adaptive virtual queue (AVQ) algorithm for active queue management. In Proc. of the ACM SIGCOMM, San Diego, California, USA, 82001.

[26] A. Lakshmikantha, C. Beck, and R. Srikant. Robustness of real and virtual queue based active queue management schemes. In Proc. of the 2003 Amer. Contr. Conf., pages 266-271, June 2003.

[27] S. H. Low. A duality model of TCP and queue management algorithms. IEEE/ACM Trans. on Networking, 11(4), Aug. 2003.

[28] S. H. Low, F. Paganini, and J. C. Doyle. Internet congestion control. IEEE Contr. Syst. Mag., 22(1): 28-43, Feb. 2002.

[29] J. Lygeros, K. H. Johansson, S. N. Simić, J. Zhang, and S. S. Sastry. Dynamical properties of hybrid automata. IEEE Trans. on Automat. Contr., 48(1):2-17, Jan. 2003.

[30] J. Lygeros, C. Tomlin, and S. Sastry. Controllers for reachability specifications for hybrid systems. Automatica, 35(3):349-370, Mar. 1999.

[31] N. A. Lynch and B. H. Krogh, editors. Hybrid Systems: Computation and Control, volume 1790 of Lect. Notes in Comput. Science. Springer, 2000.

[32] J. Mahdavi and S. Floyd. TCP-friendly unicast rate-based flow control. Technical note sent to the end2end-interest mailing list, Jan. 1997.

[33] M. Mathis, J. Semke, J. Mahdavi, and T. Ott. The macroscopic behavior of the TCP congestion avoidance algorithm. ACM Comput. Comm. Review, 27(3), July 1997.

[34] V. Misra, W. Gong, and D. Towsley. Stochastic differential equation modeling and analysis of TCPwindowsize behavior. In Proc. of PERFORMANCE'99, Istanbul, Turkey, 1999.

[35] V. Misra, W. Gong, and D. Towsley. Fluid-based analysis of a network of AQM routers supporting TCP flows with an application to RED. In Proc. of the ACM SIGCOMM, Sept. 2000.

[36] A. Nerode and W. Kohn. Models for hybrid systems: Automata, topologies, stability. In Grossman et al. [18], pages $317-356$.

[37] T. Ott, J. H. B. Kemperman, and M. Mathis. Window size behavior in TCP/IP with constant loss probability. In Proc. of the DIMACS Workshop on Performance of Realtime Applications on the Internet, Nov. 1996.

[38] J. Padhye, V. Firoiu, D. Towsley, and J. Kurose. Modeling TCP Reno performance: A simple model and its empirical validation. IEEE/ACM Trans. on Networking, 8(2):133-145, Apr. 2000.

[39] G. Pola, M. L. Bujorianu, J. Lygeros, and M. D. D. Benedetto. Stochastic hybrid models: An overview. In Proc. of the IFAC Conf. on Anal. and Design of Hybrid Syst., June 2003.

[40] A. V. D. Schaft and H. Schumacher. An Introduction to Hybrid Dynamical Systems. Number 251 in Lect. Notes in Contr. and Inform. Sci. Springer-Verlag, London, 2000.

[41] S. Shakkottai and R. Srikant. How good are deterministic fluid models of Internet congestion control? In Proc. of the IEEE INFOCOM, June 2002.

[42] B. Sikdar, S. Kalyanaraman, and K. Vastola. Analytic models for the latency and steady-state throughput of TCP Tahoe, Reno and SACK. In Proc. of the IEEE GLOBECOM, pages 25-29, 2001.

[43] B. Sikdar, S. Kalyanaraman, and K. Vastola. TCP Reno with random losses: Latency, throughput and sensitivity analysis. In Proc. of the IEEE IPCCC, pages 188-195, Apr. 2001. 
[44] L. Tavernini. Differential automata and their discrete simulators. Nonlinear Anal. Theory, Methods, and Applications, 11(6):665-683, 1987.

[45] L. Xiao and A. H. J. P. How. Control with random communication delays via a discrete-time jump system approach. In Proc. of the 2000 Amer. Contr. Conf., volume 3, pages 2199-2204, June 2000.

[46] J. Zhang, K. Johansson, J. Lygeros, and S. Sastry. Dynamical systems revisited: Hybrid systems with Zeno executions. In Lynch and Krogh [31], pages 451-464. 